\title{
CONVERGENCE AND DIVERGENCE OF KLEINIAN SURFACE GROUPS
}

\author{
JEFFREY BROCK, KENNETH BROMBERG, RICHARD CANARY AND CYRIL LECUIRE
}

\begin{abstract}
We characterize sequences of Kleinian surface groups with convergent subsequences in terms of the asymptotic behavior of the ending invariants of the associated hyperbolic 3-manifolds. Asymptotic behavior of end invariants in a convergent sequence predicts the parabolic locus of the algebraic limit as well as how the algebraic limit wraps within the geometric limit under the natural locally isometric covering map.
\end{abstract}

\section{INTRODUCTION}

Central to Thurston's original approach to the hyperbolization theorem for closed, irreducible, atoroidal 3-manifolds is a collection of compactness criteria for deformation spaces of hyperbolic 3-manifolds. In the Haken setting, such compactness results gave rise to iterative solutions to the search for hyperbolic structures on constituent pieces in a hierarchical decomposition.

Later, the classification of hyperbolic 3-manifolds with finitely generated fundamental group gave explicit a priori geometric control of these manifolds in terms of the combinatorics of the asymptotic data determining the hyperbolic structure, up to bi-Lipschitz diffeomorphism. Sullivan's Rigidity Theorem then allows for the passage from bi-Lipschitz diffeomorphism to isometry. The invariants themselves then become parameters, and the bi-Lipschitz control they provide gives rise to a new range of interrelations between geometric and topological features of the resulting manifolds.

The present paper relates these asymptotic invariants explicitly to compactness criteria, characterizing subsequential convergence precisely in terms of the invariants' limiting combinatorics vis a vis the complex of curves. In particular, we describe a manner in which invariants bound projections to curve complexes of subsurfaces, a notion that guarantees a priori bounds for geodesic lengths in a sequence. Our main theorem is a generalization of Thurston's Double Limit Theorem $([37,34])$, which provides a criterion to ensure subsequential convergence of a sequence of Kleinian surface groups, and is a key technical step in Thurston's hyperbolization theorem for 3-manifolds fibering over the circle.

Brock was partially supported by NSF grant DMS-1207572, Bromberg was partially supported by NSF grant DMS-1207873, Canary was partially supported by NSF grants DMS-1006298 and DMS -1306992, and Lecuire was partially supported by the ANR grant GDSOUS and Canary and Lecuire were partially supported by the GEAR network (NSF grants DMS-1107452, 1107263, and 1107367). 
Theorem 1.1. Let $S$ be a compact, orientable surface and let $\left\{\rho_{n}\right\}$ be a sequence in $A H(S)$ with end invariants $\left\{v_{n}^{ \pm}\right\}$. Then $\left\{\rho_{n}\right\}$ has a convergent subsequence if and only if there exists a subsequence $\left\{\rho_{j}\right\}$ of $\left\{\rho_{n}\right\}$ such that $\left\{v_{j}^{ \pm}\right\}$bounds projections.

We also (see Theorem 1.2) show that the asymptotic behavior of the end invariants predicts the curve and lamination components of the end invariants of the limit and how the algebraic limit manifold "wraps" within a geometric limit.

We briefly describe terms and notation of Theorem 1.1.

Recall that $A H(S)$ is the space of (conjugacy classes of) representations

$$
\rho: \pi_{1}(S) \rightarrow \operatorname{PSL}(2, \mathbb{C})
$$

for which $\rho$ sends peripheral elements to parabolic elements. The end invariants will be discussed more thoroughly in Section 2, but in the case that $\rho$ is quasiFuchsian, its end invariants $v^{+}(\rho)$ and $v^{-}(\rho)$ are a pair of hyperbolic structures in the Teichmüller space $\mathscr{T}(S)$. In the general setting, each end invariant $v^{ \pm}(\rho)$ is a disjoint union of a multicurve on $S$, the parabolic locus, with either an ending lamination or a complete finite-area hyperbolic structure supported on each complementary component.

Given an end invariant $v$ for $\rho$ and a curve $d$ in $\mathscr{C}(S)$, the curve complex of $S$, we define the length $l_{v}(d)$ to be 0 if $d$ is a curve in $v$, to be hyperbolic length $l_{\tau}(d)$ if $d$ lies in a subsurface $R$ admitting a complete hyperbolic structure $\tau$ induced by $\rho$, and to be $\infty$ otherwise. A collection of essential simple closed curves $\mu$ on $S$ is binding if any representative of $\mu$ on $S$ decomposes $S$ into disks or peripheral annuli. We call a fixed choice of such a collection $\mu$ a coarse basepoint for $\mathscr{C}(S)$. We define

$$
m(\nu, d, \mu)=\max \left\{\sup _{d \subset \partial Y} d_{Y}(v, \mu), \frac{1}{l_{v}(d)}\right\}
$$

where the supremum in the first term is taken over all essential subsurfaces $Y$ with $d$ contained in $\partial Y$, and the subsurface projection $d_{Y}(\nu, \mu)$ is a measure of the distance in $\mathscr{C}(Y)$ between projections $\pi_{Y}(v)$ and $\pi_{Y}(\mu)$ to $\mathscr{C}(Y)$ of $v$ and $\mu$ (see sections 2.1 and 2.2).

If we take the supremum of $d_{Y}(v, \mu)$ only over non-annular surface with boundary containing $d$ (i.e. $Y$ is not isotopic to a collar neighborhood collar $(d)$ of $d$ ), then we obtain

$$
m^{n a}(v, d, \mu)=\max \left\{\sup _{\substack{d \subset \partial Y, Y \neq \operatorname{collar}(d)}} d_{Y}(v, \mu), \frac{1}{l_{v}(d)}\right\} .
$$

Choose a coarse basepoint $\mu$ in $\mathscr{C}(S)$ once and for all. We say that a sequence $\left\{v_{n}^{ \pm}\right\}$of end invariants bounds projections if for some $K>0$ the following conditions hold:

(a) Every geodesic in $\mathscr{C}(S)$ joining $\pi_{S}\left(v_{n}^{+}\right)$to $\pi_{S}\left(v_{n}^{-}\right)$lies at distance at most $K$ from $\mu$.

(b) If $d \in \mathscr{C}(S)$ is a curve, then either 
(i) there exists $\beta(d) \in\{+,-\}$ such that $\left\{m\left(v_{n}^{\beta}, d, \mu\right)\right\}$ is eventually bounded, meaning there is $N \in \mathbb{N}$ such that

$$
\sup \left\{m\left(v_{n}^{\beta}, d, \mu\right), n \geq N\right\}<\infty,
$$

or

(ii) $\left\{m^{n a}\left(v_{n}^{+}, d, \mu\right)\right\}$ and $\left\{m^{n a}\left(v_{n}^{-}, d, \mu\right)\right\}$ are both eventually bounded and there exists $w(d) \in \mathbb{Z}$ and a sequence $\left\{s_{n}\right\} \subset \mathbb{Z}$ such that $\lim \left|s_{n}\right|=\infty$ and both

$$
\left\{d_{Y}\left(D_{Y}^{s_{n} w(d)}\left(v_{n}^{+}\right), \mu\right)\right\} \text { and }\left\{d_{Y}\left(D_{Y}^{s_{n}(w(d)-1)}\left(v_{n}^{-}\right), \mu\right)\right\}
$$

are eventually bounded when $Y=\operatorname{collar}(d)$ and $D_{Y}$ is the right Dehn-twist about $Y$.

In this definition, we say that a curve $d$ is a combinatorial parabolic if $\left\{m\left(v_{n}^{+}, d, \mu\right)\right\}$ or $\left\{m\left(v_{n}^{-}, d, \mu\right)\right\}$ is not eventually bounded. It is an upward-pointing combinatorial parabolic if $\left\{m\left(v_{n}^{+}, d, \mu\right)\right\}$ is not eventually bounded and $\left\{m\left(v_{n}^{-}, d, \mu\right)\right\}$ is eventually bounded. Similarly, we say that a curve $d$ is a downward-pointing combinatorial parabolic if $\left\{m\left(v_{n}^{-}, d, \mu\right)\right\}$ is not eventually bounded and $\left\{m\left(v_{n}^{+}, d, \mu\right)\right\}$ is eventually bounded. We say that $d$ is a combinatorial wrapped parabolic if both $\left\{m\left(v_{n}^{+}, d, \mu\right)\right\}$ and $\left\{m\left(v_{n}^{-}, d, \mu\right)\right\}$ are unbounded. If $d$ is combinatorial parabolic, then we we say that $w(d)$ is its combinatorial wrapping number.

We will see that, for a convergent sequence, every combinatorial parabolic is indeed associated to a parabolic in the limit and furthermore that one can determine which side the parabolic manifests on directly from the asymptotic behavior of $\left\{m\left(v_{n}^{+}, d, \mu\right)\right\}$ and $\left\{m\left(v_{n}^{-}, d, \mu\right)\right\}$. Moreover, every wrapped parabolic is associated to the wrapping of an immersion of a compact core for $N_{\rho}$ in a geometric limit of $\left\{N_{\rho_{n}}\right\}$.

We combine our results with [11, Theorem 1.3$]$ to see that the asymptotic behavior of the end invariants predicts the curve and lamination components of the end invariants of the limit.

We also describe, in the case when $N_{\rho_{n}}$ converges geometrically to a hyperbolic 3-manifold, how a compact core for the algebraic limit is "wrapped" when pushed down into the geometric limit. We describe this phenomenon in terms of a wrapping multicurve and an associated wrapping number (we refer the reader to section 3.1 for definitions). Anderson and Canary [1] first observed that there need not be a compact core for the algebraic limit that embeds in the geometric limit and McMullen [29, Lemma A.4] gave the first description of this phenomenon in the surface group case. We show that there is a compact core for the algebraic limit that embeds in the geometric limit if and only if the wrapping multicurve is empty.

Theorem 1.2. Suppose that $\left\{\rho_{n}\right\}$ is a sequence in $A H(S)$ converging to $\rho \in A H(S)$ and $\left\{v_{n}^{ \pm}\right\}$bounds projections. Then

(1) $\ell_{\rho}(d)=0$ if and only if $d$ is a combinatorial parabolic for the sequence $\left\{v_{n}^{ \pm}\right\}$

(2) A parabolic curve d is upward-pointing in $N_{\rho}$ if and only if

$$
\left|m\left(v_{n}^{+}, d, \mu\right)\right|-\left|m\left(v_{n}^{-}, d, \mu\right)\right| \rightarrow+\infty .
$$


(3) A lamination $\lambda \in \mathscr{E} \mathscr{L}(Y)$ is an ending lamination for an upward-pointing (respectively downward-pointing) geometrically infinite end for $N_{\rho}$ if and only if $\left\{\pi_{Y}\left(v_{n}^{+}\right)\right\}$(respectively $\left\{\pi_{Y}\left(v_{n}^{-}\right)\right\}$) converges in $\mathscr{C}(Y) \cup \mathscr{E} \mathscr{L}(Y)$ to $\lambda$.

(4) If $\left\{\rho_{n}\left(\pi_{1}(S)\right)\right\}$ converges geometrically to $\hat{\Gamma}$, then the wrapping multicurve for $\left(\left\{\rho_{n}\right\}, \rho, \hat{\Gamma}\right)$ is the collection of combinatorial wrapping parabolics given by $\left\{v_{n}^{ \pm}\right\}$and if $d$ is a wrapping parabolic, then the combinatorial wrapping number $w(d)$ agree with the actual wrapping number $w^{+}(d)$.

(5) There is a compact core for $N_{\rho}$ that embeds in $\hat{N}=\mathbf{H}^{3} / \Gamma$ if and only if there are no combinatorial wrapping parabolics.

Remark: Some results of [33] are special cases of Theorems 1.1 and 1.2.

We also obtain the following alternative characterization of convergence in terms of sequence of bounded length multicurves in $N_{\rho_{n}}$.

Theorem 1.3. Let $S$ be a compact, orientable surface and let $\left\{\rho_{n}\right\}$ be a sequence in $A H(S)$. Then $\left\{\rho_{n}\right\}$ has a convergent subsequence if and only if there exists a subsequence $\left\{\rho_{j}\right\}$ of $\left\{\rho_{n}\right\}$ and a sequence $\left\{c_{j}^{ \pm}\right\}$of pairs of multicurves so that $\left\{\ell_{\rho_{j}}\left(c_{j}^{+} \cup c_{j}^{-}\right)\right\}$is bounded and $\left\{c_{j}^{ \pm}\right\}$bounds projections.

When $c$ is a multicurve and $d$ is a curve, we define

$$
m(c, d, \mu)=\sup _{d \subset \partial Y} d_{Y}(c, \mu)
$$

if $i(c, d) \neq 0$ and $m(c, d, \mu)=\infty$ otherwise. Similarly, we define

$$
m^{n a}(c, d, \mu)=\sup _{\substack{d \subset \supset \gamma, Y \neq \operatorname{collar}(d)}} d_{Y}(c, \mu)
$$

In analogy with the end invariants situation, we say that a sequence $\left\{c_{n}^{ \pm}\right\}$of pairs of multicurves bounds projections if, choosing a coarse basepoint $\mu$ in $\mathscr{C}(S)$, the following conditions hold:

(a) every geodesic joining $\pi_{S}\left(c_{n}^{+}\right)$to $\pi_{S}\left(c_{n}^{-}\right)$lies a bounded distance from $\mu$ in $\mathscr{C}(S)$

(b) if $d \in \mathscr{C}(S)$ is a curve, then either

(i) there exists $\beta(d)$ such that $\left\{m\left(c_{n}^{\beta}, d, \mu\right)\right\}$ is eventually bounded, or

(ii) $\left\{m^{n a}\left(c_{n}^{+}, d, \mu\right)\right\}$ and $\left\{m^{n a}\left(c_{n}^{-}, d, \mu\right)\right\}$ are both eventually bounded and there exists $w(d) \in \mathbb{Z}$ and a sequence $\left\{s_{n}\right\} \subset \mathbb{Z}$ such that $\lim \left|s_{n}\right|=\infty$ and both

$$
\left\{d_{Y}\left(D_{Y}^{s_{n} w(d)}\left(c_{n}^{+}\right), \mu\right)\right\} \text { and }\left\{d_{Y}\left(D_{Y}^{s_{n}(w(d)-1)}\left(c_{n}^{-}\right), \mu\right)\right\}
$$

are eventually bounded when $Y=\operatorname{collar}(d)$ and $D_{Y}$ is the right Dehn-twist about $Y$.

We again say that a curve $d$ is an upward-pointing combinatorial parabolic if $\left\{m\left(c_{n}^{+}, d, \mu\right)\right\}$ is not eventually bounded and $\left\{m\left(c_{n}^{-}, d, \mu\right)\right\}$ is eventually bounded. Similarly, we say that a curve $d$ is a downward-pointing combinatorial parabolic if 
$\left\{m\left(c_{n}^{-}, d, \mu\right)\right\}$ is not eventually bounded and $\left\{m\left(c_{n}^{+}, d, \mu\right)\right\}$ is eventually bounded. We say that $d$ is a combinatorial wrapped parabolic if both $\left\{m\left(c_{n}^{+}, d, \mu\right)\right\}$ and $\left\{m\left(c_{n}^{-}, d, \mu\right)\right\}$ are unbounded. However, unlike in the end invariant case, the bounded length multicurves bounding projections need not predict the ending laminations or the parabolics in the algebraic limit. For example, if $\left\{\rho_{n}\right\}$ is a convergent sequence, then any constant sequence $\left\{c_{n}^{ \pm}\right\}=\left\{c^{ \pm}\right\}$of pairs of filling multicurves will bound projections. We will discuss this issue further in section 6 .

Outline of the paper: In section 2 we recall definitions and previous results that will be used in the paper. In section 3 we define the wrapping multicurve and the wrapping numbers. We assume that $\left\{\rho_{n}\right\}$ converges to $\rho$ and that $\left\{N_{\rho_{n}}\right\}$ converges geometrically to $\hat{N}$. Let $\pi: N_{\rho} \rightarrow \hat{N}$ be the obvious covering map. We first find a level surface $F$ in $N_{\rho}$ and a collection $Q$ of incompressible annuli in $F$, so that $\left.\pi\right|_{F}$ is an immersion, $\left.\pi\right|_{F-Q}$ is an embedding and $\pi$ wraps $Q$ around the boundary of a cusp region in $\hat{N}$. The collection $q$ of core curves of elements of $Q$ is the wrapping multicurve. The wrapping number then records "how many times" $Q$ is wrapped around the cusp region.

In section 4 , we prove that if a sequence $\left\{\rho_{n}\right\} \subset A H(S)$ converges, then some subsequence of its end invariants predicts convergence. We also establish Theorem 1.2. We first use work of Minsky [30, 31] and Brock-Bromberg-CanaryMinsky [11] to establish the results in the case that the wrapping multicurve is empty. When the wrapping multicurve is non-empty, we use the wrapped surface $F$ from section 3.1 to construct two new sequences, that differ from the original sequence by powers of Dehn twists in components of the wrapping multicurve, but themselves have empty wrapping multicurves. We can then apply the results from the empty wrapping multicurve case to both of these sequences. Analyzing the relationship between the end invariants of the original sequence and the two new sequences allow us to complete the proof.

In section 5, we show that if the sequence $\left\{v_{n}^{ \pm}\right\}$of end invariants for a sequence $\left\{\rho_{n}\right\}$ in $A H(S)$ bounds projections, then one can find a subsequence $\left\{\rho_{j}\right\}$ and a sequence $\left\{c_{j}^{ \pm}\right\}$of pairs of multicurves such that $\left\{\ell_{\rho_{j}}\left(c_{j}^{+} \cup c_{j}^{-}\right)\right\}$is bounded and $\left\{c_{j}^{ \pm}\right\}$ bounds projections. The difficulty comes from the fact that one must insure that $c_{n}^{+}$ and $c_{n}^{-}$do not share any curves while bounding projections. In particular one must take special care of the curves where $\left\{m\left(v_{n}^{\beta}, d, \mu\right)\right\}$ is unbounded. To overcome these difficulties, we will construct $c_{n}^{ \pm}$as minimal length pants decompositions under some constraints.

In section 6, we show that if $\left\{\rho_{n}\right\}$ is a sequence in $A H(S)$ and there is a sequence of bounded length multicurves $\left\{c_{n}^{ \pm}\right\}$that bound projections, then $\left\{\rho_{n}\right\}$ has a convergent subsequence. Again we start with the case that the wrapping multicurve is empty. We may assume that each $c_{n}^{ \pm}$is a pants decomposition of $S$. We first use results of Minsky [30] to find a pants decomposition $r$ such that $\left\{\ell_{\rho_{n}}(r)\right\}$ is bounded. We then construct the model manifold $M_{n}^{\beta}$ associated to the hierarchy joining $r$ to $c_{n}^{\beta}$ and observe, using work of Bowditch [9] and Minsky [31], that there is a uniformly Lipschitz map of $M_{n}^{\beta}$ into $N_{\rho_{n}}$. (If $r$ and $c_{n}^{\beta}$ share curves 
we consider a model manifold associated to a subsurface of $S$.) We find a bounded length transversal in $M_{n}$ to each curve in $r$ and then observe that it also has bounded length in $N_{\rho_{n}}$. We pass to a subsequence so that the sequence of transversals we have constructed is constant and then simply apply the Double Limit Theorem to conclude that there is a convergent subsequence. When the wrapping multicurve is not empty, we construct two new sequences with empty wrapping multicurves and use them to produce a converging subsequence of the original sequence.

Finally, in section 7 we combine the results of sections 4,5 and 6 to complete the proofs of both Theorems 1.1 and 1.3.

\section{BACKGROUND}

In this section, we collect definitions and previous results which will be used in the paper. We first need to recall the definitions of curve complexes of subsurfaces, subsurface projections, markings and end invariants.

2.1. Curve complexes, markings and subsurface projections. If $W$ is an essential non-annular subsurface of $S$, its curve complex $\mathscr{C}(W)$ is a locally infinite simplicial complex whose vertices are isotopy classes of essential non-peripheral curves on $W$. Two vertices are joined by an edge if and only if the associated curves intersect minimally. A collection of $n+1$ vertices span a $n$-simplex if the corresponding curves have mutally disjoint representatives. Masur and Minsky [26] proved that $\mathscr{C}(W)$ is Gromov hyperbolic with respect to its natural path metric.

We will assume throughout that all curves are essential and non-peripheral. A multicurve will be a collection of disjoint curves, no two of which are homotopic. A pants decomposition of $W$ is a maximal multicurve.

Klarreich [22], see also Hamenstadt [18], showed that the Gromov boundary $\partial_{\infty} \mathscr{C}(W)$ of $\mathscr{C}(W)$ can be naturally identified with the space $\mathscr{E} \mathscr{L}(W)$ of filling geodesic laminations on $W$.

A marking $\mu$ on $S$ is a multicurve base $(\mu)$ together with a selection of transversal curves, at most one for each component of base $(\mu)$. A transversal curve to a curve $c$ in base $(\mu)$ intersects $c$ and is disjoint from base $(\mu)-c$. A marking is complete if base $(\mu)$ is a pants decomposition and every curve in base $(\mu)$ has a transversal. A generalized marking is a collection of filling laminations on a disjoint collection of subsurfaces together with the boundary of those subsurfaces and a marking of their complement. (See Masur-Minsky [27] and Minsky [31] for a more careful discussion of markings and generalized markings.)

If $W$ is an essential non-annular subsurface, one may define a subsurface projection

$$
\pi_{W}: \mathscr{C}(S) \rightarrow \mathscr{C}(W) \cup\{\emptyset\} .
$$

If $c \in \mathscr{C}(S)$ and $c$ is disjoint from $W$, then $\pi_{W}(c)=\emptyset$. If not, $c \cap W$ is a collection of arcs and curves on $W$. Each arc in $c \cap W$ may be surgered to produce an essential curve on $W$ by adding arcs in $\partial W$. We let $\pi_{W}(c)$ denote a choice of one of the resulting essential curves in $W$; then $\pi_{W}(c)$ is coarsely well-defined - any two choices lie at bounded distance (see [27, Lemma 2.3]). For a subset of $\mathscr{C}(S)$ (such 
as a multicurve, a marking or a coarse basepoint for $\mathscr{C}(S)$ ), we choose $\pi_{W}(\mu)$ to be a curve in $\bigcup_{c \in \mu} \pi_{W}(\mu)$ if there is one and to be $\emptyset$ otherwise. We can then define

$$
d_{W}(c, d)=d_{\mathscr{C}(W)}\left(\pi_{W}(c), \pi_{W}(d)\right)
$$

if $\pi_{W}(c) \neq \emptyset$ and $\pi_{W}(d) \neq \emptyset$, and define $d_{W}(c, d)=+\infty$ otherwise.

If $\mu$ is a generalized marking on $S$, then we define

$$
\pi_{W}(\mu) \in \mathscr{C}(W) \cup \mathscr{E} \mathscr{L}(W) \cup \emptyset
$$

by

(1) letting $\pi_{W}(\mu)=\emptyset$ if $\mu$ does not intersect $W$,

(2) letting $\pi_{W}(\mu)=\lambda$ if $\lambda \subset \mu$ lies in $\mathscr{E} \mathscr{L}(W)$,

(3) constructing $\pi_{W}(\mu)$ as above using any simple closed curve or proper arc in $\mu \cap W$.

For a pair of generalized markings, we define

$$
d_{W}\left(\mu, \mu^{\prime}\right)=d_{\mathscr{C}(W)}\left(\pi_{W}(\mu), \pi_{W}\left(\mu^{\prime}\right)\right)
$$

if $\pi_{W}(\mu), \pi_{W}\left(\mu^{\prime}\right) \in \mathscr{C}(W)$ and $d_{W}\left(\mu^{\prime}, \mu\right)=\infty$ if $\pi_{W}(\mu)$ or $\pi_{W}\left(\mu^{\prime}\right)$ lies in $\mathscr{E} \mathscr{L}(W) \cup\{\emptyset\}$.

If $W$ is an essential annulus in $S$ we may also define $d_{W}(c, d)$ and $d_{W}(c, \mu)$. The simplest way to do this is to first fix a hyperbolic metric on $S$ and let $\tilde{S}$ be the annular cover $S$ so that $W$ lifts to a compact core for $\tilde{S}$. We then compactify $\tilde{S}$ by its ideal boundary to obtain an annulus $A$ and define a complex $\mathscr{C}(W)$ whose vertices are geodesics in $A$ that joins the two boundary components of $A$. We join two vertices if they have disjoint representatives. If we give $\mathscr{C}(W)$ the natural path metric then $d_{\mathscr{C}(W)}(a, b)=i(a, b)+1$ and it follows that $\mathscr{C}(W)$ is quasi-isometric to $\mathbb{Z}$. Given a simple closed curve $c \subset S$, we realize it as geodesic and then consider its pre-image $\tilde{c}$ in $\tilde{S}$. If $\tilde{c}$ contains an essential arc $\alpha$ whose closure joins the two boundary components of $A$, we set $\pi_{W}(c)=\alpha$ and we set $\pi_{W}(c)=\emptyset$ otherwise. For a multicurve or marking $\mu$, we define $\pi_{W}(\mu)$ to be an element of $\bigcup_{c \in \mu} \pi_{W}(\mu)$. We can then define

$$
d_{W}(c, d)=d_{\mathscr{C}(W)}(\tilde{c}, \tilde{d})
$$

if $\pi_{W}(c) \neq \emptyset$ and $\pi_{W}(d) \neq \emptyset$, and define $d_{W}(c, d)=+\infty$ otherwise. One can check that this definition is independent of the choice of metric. (Again see MasurMinsky [27] and Minsky [31] for a complete discussion of subsurface projections and the resulting distances.)

In all cases, the distance between two curves (or markings) is bounded above by a function of their intersection number.

Lemma 2.1. ([26, Lemma 2.1]) If $S$ is a compact orientable surface, $\alpha, \beta$ are multicurves or markings on $S$ and $W$ is an essential subsurface of $S$, then

$$
d_{W}(\alpha, \beta) \leq 2 i(\alpha, \beta)+1 .
$$

The following estimate is often useful in establishing relationships between subsurface projections. Behrstock ([5, Theorem 4.3]) first gave a version with inexplicit constants which depends on the surface $S$. We will use a version, due to Leininger, with explicit universal constants. 
Lemma 2.2. ([24, Lemma 2.13]) Given a compact surface $S$, two essential subsurfaces $Y$ and $Z$ which overlap and a generalized marking $\mu$ which intersects both $Y$ and $Z$, then

$$
d_{Y}(\mu, \partial Z) \geq 10 \Longrightarrow d_{Z}(\mu, \partial Y) \leq 4
$$

We will also use the fact that a sequence of curves which is not eventually constant blows up on some subsurface.

Lemma 2.3. Given a sequence of simple closed curves $\left\{c_{n}\right\}$ and a complete marking $\mu$ on a compact surface $S$, there is a subsequence $\left\{c_{j}\right\}$ such that either $\left\{c_{j}\right\}$ is constant or there is a subsurface $Y \subseteq S$ with $d_{Y}\left(\mu, c_{j}\right) \longrightarrow \infty$.

Proof. Fix a metric on $S$ and realize the sequence $\left\{c_{n}\right\}$ as a sequence of closed geodesics. We then extract a subsequence $\left\{c_{j}\right\}$ that converges in the Hausdorff topology on closed subsets of $S$ to a geodesic lamination $\lambda$. If $\lambda$ contains an isolated simple closed curve, then $\left\{c_{j}\right\}$ is eventually constant and we are done. If not, let $Y$ be the supporting subsurface of a minimal sublamination $\lambda_{0}$ of $\lambda$. If $Y$ is not an annulus, then $\lambda_{0} \in \mathscr{E} \mathscr{L}(S)$ and results of Klarreich [22, Theorem 1.4] (see also Hamenstadt [18]) imply that $d_{Y}\left(\mu, c_{j}\right) \rightarrow \infty$.

If $\lambda_{0}$ is a simple closed geodesic, then $Y=\operatorname{collar}\left(\lambda_{0}\right)$ is an annulus and, since $\lambda$ doesn't contain an isolated simple close curve, there must be leaves of $\lambda$ spiraling around $\lambda_{0}$. Let $\tilde{S}_{0}$ be the annular cover of $S$ associated to the cyclic subgroup of $\pi_{1}(S)$ generated by $\lambda_{0}$ and let $\tilde{\lambda}_{0}$ be the unique lift of $\lambda_{0}$ to $\tilde{S}_{0}$. Let $\tilde{c}_{j}=\pi_{Y}\left(c_{j}\right)$. Since $\left\{c_{j}\right\}$ converges to $\lambda$ in the Hausdorff topology and there exist leaves of $\lambda$ spiraling about $\lambda_{0}$, the acute angle between $\tilde{c}_{j}$ and $\tilde{\lambda}_{0}$ converges to 0 . It follows that $i\left(\tilde{c}_{j}, \tilde{a}\right) \rightarrow \infty$ for any fixed element $\tilde{a} \in \mathscr{C}(Y)$. In particular, if $d$ is a component of $\mu$ that intersects $\lambda_{0}$ and $\tilde{d}=\pi_{Y}(d)$, then

$$
d_{Y}\left(c_{j}, d\right)=d_{\mathscr{C}(Y)}\left(\tilde{c}_{j}, \tilde{d}\right)=i\left(\tilde{c}_{j}, \tilde{d}\right)+1 \rightarrow \infty .
$$

It follows that $d_{Y}\left(c_{j}, \mu\right) \rightarrow \infty$ as desired.

2.2. End invariants. If $\rho \in A H(S)$, the end invariants of $N_{\rho}$ encode the asymptotic geometry of $N_{\rho}=\mathbf{H}^{3} / \rho\left(\pi_{1}(S)\right)$. The Ending Lamination Theorem (see Minsky [31] and Brock-Canary-Minsky [12]) asserts that a representation $\rho \in A H(S)$ is uniquely determined by its end invariants. The reader will find a more extensive discussion of the definition of the end invariants and the Ending Lamination Theorem in Minsky [31].

A $\rho\left(\pi_{1}(S)\right.$ )-invariant collection $\mathscr{H}$ of disjoint horoballs in $\mathbf{H}^{3}$ is a precisely invariant collection of horoballs for $\rho\left(\pi_{1}(S)\right)$ if there is a horoball based at the fixed point of every parabolic element of $\rho\left(\pi_{1}(S)\right.$ ) (and every horoball in $\mathscr{H}$ is based at a parabolic fixed point). The existence of such a collection is a classical consequence of the Margulis Lemma, see [25, Proposition VI.A.11] for example. We define

$$
N_{\rho}^{0}=\left(\mathbf{H}^{3}-\bigcup_{H \in \mathscr{H}} H\right) / \rho\left(\pi_{1}(S)\right)
$$


If $\mathscr{H}_{p}$ denotes the set of horoballs in $\mathscr{H}$ which are associated to peripheral elements of $\pi_{1}(S)$, then we define

$$
N_{\rho}^{1}=\left(\mathbf{H}^{3}-\bigcup_{H \in \mathscr{H}_{p}} H\right) / \rho\left(\pi_{1}(S)\right) .
$$

A relative compact core for $N_{\rho}^{0}$ is a compact submanifold $M_{\rho}$ of $N_{\rho}^{0}$ such that the inclusion of $M_{\rho}$ into $N_{\rho}$ is a homotopy equivalence and $M_{\rho}$ intersects each component of $\partial N_{\rho}^{0}$ in an incompressible annulus. Let $P_{\rho}=M_{\rho} \cap \partial N_{\rho}^{0}$ and let $P_{\rho}^{1}=M_{\rho} \cap \partial N_{\rho}^{1}$. (See Kulkarni-Shalen [23] and McCullough [28] for proofs that $N_{\rho}^{0}$ admits a relative compact core.)

Bonahon [8] showed that there is an orientation preserving homeomorphism from $S \times \mathbb{R}$ to $N_{\rho}^{1}$ in the homotopy class determined by $\rho$. We will implicitly identify $N_{\rho}^{1}$ with $S \times \mathbb{R}$ throughout the paper. Suppose that $W$ is a subsurface of $S$ and $f: W \rightarrow N_{\rho}^{1}$ is a map of $W$ into $S \times \mathbb{R}$ (in the homotopy class associated to $\left.\rho\right|_{\pi_{1}(W)}$ ). We say that $f$ (or $f(W)$ ) is a level subsurface if it is an embedding which is isotopic to $W \times\{0\}$. If $W=S$, we say $f$ (or $f(S)$ ) is a level surface.

The conformal boundary $\partial_{c} N_{\rho}$ of $N_{\rho}$ is the quotient by $\Gamma$ of the domain $\Omega(\rho)$ of discontinuity for the action of $\rho(\Gamma)$ on $\hat{\mathbb{C}}$. One may identify the conformal boundary $\partial_{c} N_{\rho}$ with a collection of components of $\partial M_{\rho}-P_{\rho}$. The other components of $\partial M_{\rho}-P_{\rho}$ bound neighborhoods of geometrically infinite ends of $N_{\rho}^{0}$. If $E$ is a geometrically infinite end with a neighborhood bounded by a component $W$ of $\partial M_{\rho}-P_{\rho}$, then there exists a sequence $\left\{\alpha_{n}\right\} \subset \mathscr{C}\left(W, \rho, L_{1}\right)$, for some $L_{1}=L_{1}(S)>0$, whose geodesic representatives $\left\{\alpha_{n}^{*}\right\}$ exit $E$ (see Lemma 2.9 for a more careful statement). The sequence $\left\{\alpha_{n}\right\}$ converges to an ending lamination $\lambda \in \mathscr{E} \mathscr{L}(S)$ and we call $\lambda$ the ending lamination of $E$ ( $\lambda$ does not depend on the choice of the sequence $\left.\left\{\alpha_{n}\right\}\right)$. Moreover, if $\left\{\beta_{n}\right\}$ is any sequence in $\mathscr{C}(W)$ which converges to $\lambda$, then the sequence $\left\{\beta_{n}^{*}\right\}$ of geodesic representatives in $N_{\rho}$ exits $E$. (See Bonahon [8] for an extensive discussion of geometrically infinite ends.)

There exists an orientation-preserving homeomorphism of $S \times I$ with $M_{\rho}$, again in the homotopy class determined by $\rho$, so that $\partial S \times I$ is identified with $P_{\rho}^{1}$. Let $P_{\rho}^{+}$denote the components of $P_{\rho}$ contained in $S \times\{1\}$ and let $P_{\rho}^{-}$denote the component of $P_{\rho}$ contained in $S \times\{0\}$. A core curve of a component of $P_{\rho}^{+}$is called an upward-pointing parabolic curve and a core curve of a component of $P_{\rho}^{-}$is called a downward-pointing parabolic curve. Similarly, a component of $\partial_{c} N_{\rho}$ or a geometrically infinite end of $N_{\rho}$ is called upward-pointing if it is identified with a a subsurface of $S \times\{1\}$, and is called downward-pointing if it is identified with a subset of $S \times\{0\}$.

The end invariant $v_{\rho}^{+}$consists of the multicurve $p^{+}$of upward-pointing parabolic curves together with a conformal structure on each geometrically finite component of $S \times\{1\}-p^{+}$, coming from the conformal structure on the associated component of the conformal boundary, and a filling lamination on each geometrically infinite component, which is the ending lamination of the associated end. The end invariant $v_{\rho}^{-}$is defined similarly. 
If $v$ is an end invariant, we define an associated generalized marking $\mu(v)$. We let base $(\mu(v))$ consist of all the curve and lamination components of $v$ together with a minimal length pants decomposition of the conformal (hyperbolic) structure on each geometrically finite component. For each curve in the minimal length pants decomposition of a geometrically finite component we choose a minimal length transversal. Notice that the associated marking is well-defined up to uniformly bounded ambiguity.

Given $\rho \in A H(S)$ with end invariants $v^{ \pm}$, we then define, for each essential subsurface $W$ of $S$,

$$
\pi_{W}\left(v^{ \pm}\right)=\pi_{W}\left(\mu\left(v^{ \pm}\right)\right) .
$$

Property (3) in Theorem 1.2 can be viewed as a continuity property for the projections of end invariants to subsurfaces. This property was established by BrockBromberg-Canary-Minsky in [11]:

Theorem 2.4. ([11, Theorem 1.1]) Let $\rho_{n} \longrightarrow \rho$ in $A H(S)$. If $W \subseteq S$ is an essential subsurface of $S$, other than an annulus or a pair of pants, and $\lambda \in E L(W)$ is a lamination supported on $W$, then the following statements are equivalent :

(1) $\lambda$ is a component of $v_{\rho}^{+}$.

(2) $\left\{\pi_{W}\left(v_{\rho_{n}}^{+}\right)\right\}$converges to $\lambda$.

2.3. The bounded length curve set. The Ending Lamination Theorem [31, 12] assures that the end invariants coarsely determine the geometry of $N_{\rho}$. In particular, one can use the end invariants to bound the lengths of curves in $N_{\rho}$ and to coarsely determine the set of curves of bounded length. We will need several manifestations of this principle.

It is often useful to, given $L>0$, consider the set of all curves in $N_{\rho}$ with length at most $L$. We define

$$
\mathscr{C}(\rho, L)=\left\{d \in \mathscr{C}(S) \mid \ell_{\rho}(d) \leq L\right\} .
$$

Minsky, in [30], showed that if the projection of $\mathscr{C}(\rho, L)$ to $\mathscr{C}(W)$ has large diameter, then $\partial W$ is short in $N_{\rho}$.

Theorem 2.5. ([30, Theorem 2.5]) Given $S, \varepsilon>0$ and $L>0$, there exists $B(\varepsilon, L)$ such that if $\rho \in A H(S), W \subset S$ is a proper subsurface and

$$
\operatorname{diam}\left(\pi_{W}(\mathscr{C}(\rho, L))\right)>B(\varepsilon, L),
$$

then $l_{\tau}(\partial W)<\varepsilon$.

In [11] it is proven that $\pi_{W}(\mathscr{C}(\rho, L))$ is well-approximated by a geodesic joining $\pi_{W}\left(v^{+}\right)$to $\pi_{W}\left(v^{-}\right)$.

Theorem 2.6. ([11, Theorem 1.2]) Given $S$, there exists $L_{0}>0$ such that for all $L \geq L_{0}$, there exists $D_{0}=D_{0}(L)$, such that, if $\rho \in A H(S)$ has end invariants $v^{ \pm}$, and $W \subset S$ is an essential subsurface more complicated than a thrice-punctured sphere, then $\pi_{W}(C(\rho, L))$ has Hausdorff distance at most $D_{0}$ from any geodesic in $\mathscr{C}(W)$ joining $\pi_{W}\left(v^{+}\right)$to $\pi_{W}\left(v^{-}\right)$. Moreover, if $d_{W}\left(v^{+}, v^{-}\right)>D_{0}$, then

$$
C(W, \rho, L)=\left\{\alpha \in \mathscr{C}(W): l_{\alpha}(\rho)<L\right\}
$$


is nonempty and also has Hausdorff distance at most $D_{0}$ from any geodesic in $\mathscr{C}(W)$ joining $\pi_{W}\left(v^{+}\right)$to $\pi_{W}\left(v^{-}\right)$.

As a generalization of Minsky's a priori bounds (see [31, Lemma 7.9]), Bowditch proved that all curves on a tight geodesic in $\mathscr{C}(W)$ joining two bounded length multicurves, also have bounded length. We recall that if $W$ is a non-annular essential subsurface of $S$, then a tight geodesic is a sequence $\left\{w_{i}\right\}$ of simplices in $\mathscr{C}(W)$ such that if $v_{i}$ is a vertex of $w_{i}$ and $v_{j}$ is a vertex of $w_{j}$, then $d_{W}\left(v_{i}, v_{j}\right)=|i-j|$ and each $w_{i}$ is the boundary of the subsurface filled by $w_{i-1} \cup w_{i+1}$.

Theorem 2.7. (Bowditch [9, Theorem 1.3]) Let $S$ be a compact orientable surface. Given $L>0$ there exists $R(L, S)$ such that if $\rho \in A H(S), W$ is an essential nonannular subsurface of $S,\left\{w_{i}\right\}_{i=0}^{n}$ is a tight geodesic in $\mathscr{C}(W)$, and $\ell_{\rho}\left(w_{0}\right) \leq L$ and $\ell_{\rho}\left(w_{n}\right) \leq L$, then

$$
\ell_{\rho}\left(w_{i}\right) \leq R
$$

for all $i=1, \ldots, n-1$.

2.4. Margulis regions and topological ordering. There exists a constant $\varepsilon_{3}>0$, known as the Margulis constant, such that if $\varepsilon \in\left(0, \varepsilon_{3}\right)$ and $N$ is a hyperbolic 3-manifold, then each component of the thin part

$$
N_{\text {thin }(\varepsilon)}=\left\{x \in N \mid \operatorname{inj}_{N}(x)<\varepsilon\right\}
$$

is either a solid torus neighborhood of a closed geodesic or the quotient of a horoball by a group of parabolic elements (see [36, Corollary 5.10.2] for example). If $\rho \in A H(S)$ and $d$ is a curve on $S$, then let $\mathbb{T}_{\varepsilon}(d)$ be the component of $N_{\text {thin }(\varepsilon)}$ whose fundamental group is generated by $d$. With this definition, $\mathbb{T}_{\varepsilon}(d)$ will often be empty. When it is non-empty, we will call it a Margulis region and when it is non-compact we will call it a Margulis cusp region. Notice that if $N=\mathbf{H}^{3} / \Gamma$, then the pre-image in $\mathbf{H}^{3}$ of all the non-compact components of $N_{\operatorname{thin}(\varepsilon)}$, for any $\varepsilon \in\left(0, \varepsilon_{3}\right)$, is a precisely invariant system of horoballs for $\Gamma$.

Suppose that $\alpha$ and $\beta$ are homotopically non-trivial curves in $N_{\rho}^{1}$ and that their projections to $S$ intersect essentially. We say that $\alpha$ lies above $\beta$ if $\alpha$ may be homotoped to $+\infty$ in the complement of $\alpha$ (i.e. $\alpha$ may be homotoped into $S \times[R, \infty$ ) in the complement of $\beta$ for all $R$ ). Similarly, we say that $\beta$ is below $\alpha$ if $\beta$ may be homotoped to $-\infty$ in the complement of $\alpha$ (see $[11, \S 2.5]$ for a more detailed discussion).

It is shown in [11] that if the geodesic representative of a curve $d$ lies above the geodesic representative of the boundary component of a subsurface $W$, then the projection of $d$ lies near the projection of $v^{+}$.

Theorem 2.8. ([11, Theorem 1.3] ) Given $S$ and $L>0$ there exists $D=D(S, L)$ such that if $\alpha \in \mathscr{C}(S), \rho \in A H(S)$ has end invariants $v^{ \pm}, l_{\rho}(\alpha)<L, \alpha$ overlaps a proper subsurface $W \subset S$ (other than a thrice-punctured sphere), and there exists a component $\beta$ of $\partial W$ such that $\alpha^{*}$ lies above $\beta^{*}$ in $N_{\rho}$, then

$$
d_{W}\left(\alpha, v^{+}\right)<D
$$


Remark: If $\rho(\alpha)$ is parabolic, then $\alpha$ has no geodesic representative in $N_{\rho}$. If $\alpha$ is an upward-pointing parabolic, it is natural to say that it lies above the geodesic representative of every curve it overlaps, while if $\alpha$ is a downward-pointing parabolic, it is natural to say that it lies below the geodesic representative of every curve it overlaps.

The following observation is a consequence of the geometric description of geometrically infinite ends (see Bonahon [8]).

Lemma 2.9. Given a compact surface $S$, there exists $L_{1}=L_{1}(S)$ such that if $\rho \in A H(S), W$ is an essential sub-surface of $S$ which is the support of a geometrically infinite end $E$ of $N_{\rho}^{0}$ and $\Delta$ is a finite subset of $\mathscr{C}(W)$, then there exists a pants decomposition $r$ of $W$ such that $l_{\rho}(r) \leq L_{1}$ and any curve in $r$ lies above, respectively below, any curve in $\Delta$ when $E$ is upward-pointing, respectively downward-pointing.

2.5. Lipschitz surfaces and bounded length curves. If $\rho \in A H(S)$, then a $K$-Lipschitz surface in $N_{\rho}$ is a $\pi_{1}$-injective $K$-Lipschitz map $f: X \rightarrow N_{\rho}$ where $X$ is a (complete) finite area hyperbolic surface. Incompressible pleated surfaces, see Thurston [36, section 8.8] and Canary-Epstein-Green [15, Chapter I.5], are examples of 1-Lipschitz surfaces. If $W$ is an essential subsurface of $S$ and $\alpha \in \mathscr{C}(W)$, then we say that a $K$-Lipschitz surface $f: X \rightarrow N_{\rho}$, where $X$ is a hyperbolic structure on $\operatorname{int}(W)$, realizes the pair $(\alpha, W)$ if there exists a homeomorphism $h: \operatorname{int}(W) \rightarrow X$ such that $(f \circ h)_{*}$ is conjugate to $\left.\rho\right|_{\pi_{1}(W)}$ and $f(h(\alpha))=\alpha^{*}$. Thurston observed that if $\rho\left(\pi_{1}(\partial W)\right)$ is purely parabolic and $\rho(\alpha)$ is hyperbolic, then one may always find a pleated surface realizing $(\alpha, W)$.

Lemma 2.10. (Thurston [36, Section 8.10], Canary-Epstein-Green [15, Theorem I.5.3.6]) Suppose that $\rho \in A H(S), W$ is an essential subsurface of $S$ and $\alpha \in \mathscr{C}(W)$. If every (non-trivial) element of $\rho\left(\pi_{1}(\partial W)\right)$ is parabolic and $\rho(\alpha)$ is hyperbolic, then there exists a 1-Lipschitz surface realizing $(\alpha, W)$.

One may use Lemma 2.10 and a result of Bers ([7], see also [14, p.123]) to construct bounded length pants decompositions which include any fixed bounded length curve.

Lemma 2.11. Suppose that $\rho \in A H(S), W$ is an essential subsurface of $S$ and $\alpha \in \mathscr{C}(W)$. Given $L>0$, there is $L^{\prime}=L^{\prime}(L, S)$ such that, if $\ell_{\rho}(\alpha)+\ell_{\rho}(\partial W) \leq L$, then $W$ admits a pants decomposition $p$ containing $\alpha$ such that $\ell_{\rho}(p) \leq L^{\prime}$.

2.6. Geometric limits. A sequence $\left\{\Gamma_{n}\right\}$ of Kleinian groups converges geometrically to a Kleinian group $\hat{\Gamma}$ if every accumulation point $\gamma$ of every sequence $\left\{\gamma_{n} \in \Gamma_{n}\right\}$ lies in $\hat{\Gamma}$ and if every element $\alpha$ of $\Gamma_{\infty}$ is the limit of a sequence $\left\{\alpha_{n} \in \Gamma_{n}\right\}$. It is useful, to think of geometric convergence of a sequence of torsion-free Kleinian groups, in terms of geometric convergence of the sequence of hyperbolic 3-manifolds. The following result combines standard results about geometric convergence which will be used in the paper. 
Lemma 2.12. Suppose that $\left\{\rho_{n}: \pi_{1}(S) \rightarrow \operatorname{PSL}(2, \mathbb{C})\right\}$ is a sequence of discrete faithful representations converging to the discrete faithful representation $\rho: \pi_{1}(S) \rightarrow$ $\operatorname{PSL}(2, \mathbb{C})$. Then, there exists a subsequence $\left\{\rho_{j}\right\}$ so that $\left\{\rho_{j}\left(\pi_{1}(S)\right)\right\}$ converges geometrically to $\hat{\Gamma}$.

Let $\hat{N}=\mathbb{H}^{3} / \hat{\Gamma}$ and let $\pi: N_{\rho} \rightarrow \hat{N}$ be the natural covering map. Let $\hat{\mathscr{H}}$ be a precisely invariant system of horoballs for $\hat{\Gamma}$.

There exists a nested sequence $\left\{Z_{j}\right\}$ of compact sub-manifolds exhausting $\hat{N}$ and $K_{j}$-bilipschitz smooth embeddings $\psi_{j}: Z_{j} \rightarrow N_{\rho_{j}}$ such that:

(1) $K_{j} \rightarrow 1$.

(2) If $V$ is a compact component of $\partial \hat{N}^{0}$, then, for all large enough $n, \psi_{j}(\partial V)$ is the boundary of a Margulis region for $N_{\rho_{j}}$.

(3) If $Q$ is a compact subset of a non-compact component of $\partial \hat{N}^{0}$, then, for all large enough $j, \psi_{j}(Q)$ is contained in the boundary of a Margulis region $V_{j}$ for $N_{\rho_{j}}$ and $\psi_{j}\left(Z_{j} \cap \hat{N}^{0}\right)$ does not intersect $V_{j}$.

(4) If $X$ is a finite complex and $h: X \rightarrow N_{\rho}$ is continuous, then, for all large enough $j,\left(\psi_{j} \circ \pi \circ h\right)_{*}$ is conjugate to $\rho_{j} \circ \rho^{-1} \circ h_{*}$.

Proof. The existence of the subsequence $\left\{\rho_{j}\right\}$ is guaranteed by Canary-EpsteinGreen [15, Thm. 3.1.4]. The existence of the sub-manifolds $\left\{Z_{n}\right\}$ and the comparison maps $\left\{\psi_{n}\right\}$ with property (1) is given by [15, Thm. 3.2.9 ]. Properties (2) and (3) are obtained by Brock-Canary-Minsky [12, Lemma 2.8]. Property (4) is observed in [12, Prop. 2.7], see also Anderson-Canary [2, Lemma 7.2].

\section{THE WRAPPING MULTICURVE}

In this section, we analyze how compact cores for algebraic limits immerse into geometric limits. We will see that if $\left\{\rho_{n}\right\} \subset A H(S)$ converges algebraically to $\rho$ and $\left\{\rho_{n}\left(\pi_{1}(S)\right)\right\}$ converges geometrically to $\hat{\Gamma}$, then there is a level surface $F \subset N_{\rho}^{0}$ and a collection $Q$ of incompressible annuli in $F$ so that the covering map $\pi$ : $N_{\rho}^{0} \rightarrow \hat{N}^{0}$ is an embedding on $F-Q$ and (non-trivially) wraps each component of $Q$ around a toroidal component of $\partial \hat{N}_{\rho}^{0}$. The collection $q$ of core curves of $Q$ is called the wrapping multicurve and we will define a wrapping number associated to each component of $q$ which records how many times the surface wraps the associated annulus around the toroidal component of $\partial \hat{N}_{\rho}^{0}$.

3.1. Wrapped surfaces. We first examine the topology of the situation. Given a compact non-annular surface $G$ and $e \in \mathscr{C}(G)$, let $E=\operatorname{collar}(e)$ be an open collar neighborhood of $e$ on $G, \hat{G}=G-E$,

$$
X=G \times[-1,1] \text { and } \hat{X}=X-V \text { where } V=E \times\left(-\frac{1}{2}, \frac{1}{2}\right) \subset X
$$

is a solid torus in the homotopy class of $e$. If $T=\partial V$ and $\hat{Z}=\hat{G} \times\{0\} \cup T$, then $\hat{Z}$ is a spine for $\hat{X}$. An orientation on $G$ determines an orientation on $X$ and hence on $V$ which induces an orientation on $T$. Let $m$ be an essential curve on $T$ that bounds a disk in $V$ and let $l$ be one of the components of $\partial \bar{E} \times\{0\}$. We orient this meridian 
and longitude so that the orientation of $(m, l)$ agrees with the orientation of $T$. We also decompose $T$ into two annuli with

$$
A=\partial \bar{E} \times[0,1 / 2] \cup E \times\{1 / 2\} \text { and } B=\partial \bar{E} \times[-1 / 2,0] \cup E \times\{-1 / 2\} .
$$

We will show that every map from $G$ to $\hat{X}$ that is homotopic, in $X$, to a level inclusion, is homotopic, in $X$ to exactly one of a family $\left\{f_{k}: G \rightarrow \hat{X}\right\}_{k \in \mathbb{Z}}$ of standard wrapping maps. Let $f_{1}: G \rightarrow X$ be an embedding such that the restriction of $f_{1}$ to $\hat{G}$ is $i d \times\{0\}$, i.e. $f_{1}(x)=(x, 0)$ if $x \in \hat{G}$, and $\left.f_{1}\right|_{\bar{E}}$ is a homeomorphism to $A$. For all $k \in \mathbb{Z}$, let $\phi_{k}: T \rightarrow T$ be an immersion which is the identity on $B$ and wraps $A$ "k times around" $T$, namely $\left(\phi_{k}\right)_{*}(m)=k m$ and $\left(\phi_{k}\right)_{*}(l)=l$. We then define $f_{k}: G \rightarrow \hat{Z} \subset X$ by $\left.f_{k}\right|_{\hat{G}}=\left.f_{1}\right|_{\hat{G}}$ and $\left.f_{k}\right|_{\bar{E}}=\left.\phi_{k} \circ f_{1}\right|_{\bar{E}}$. Note that all of these maps are homotopic as maps to $X$. As maps to $\hat{Z}$ (or $\hat{X}$ ) they are homotopically distinct as can be seen by counting the algebraic intersection with a point on $A$ and a point on $B$. We will call $k$ the wrapping number of $f_{k}$.

The next lemma allows us to define a wrapping number for any map in the correct homotopy class.

Lemma 3.1. Let $g:(G, \partial G) \rightarrow(\hat{X}, \partial G \times[-1,1])$ be a map such that $g$ is homotopic to id $\times\{0\}$, as a map into $(X, \partial G \times[-1,1])$. Then there exists a unique $k \in \mathbb{Z}$ such that $g$ is homotopic to $f_{k}$ as a map into $(\hat{X}, \partial G \times[-1,1])$.

Proof. Since $\hat{Z}$ is a spine of $\hat{X}$, we may assume that the image of $g$ lies in $\hat{Z}$. Since $g$ is homotopic to the level inclusion $i d \times\{0\}$ on $\hat{G}$, we may homotope $g$ within $\hat{X}$ so that $g$ agrees with the level inclusion on $\hat{G}$. Since every immersed incompressible annulus in $\hat{X}$ with boundary in $T$ is homotopic, rel boundary, into $T$, we can further homotope $g$, rel $\hat{G}$, such that $g(E) \subset T$. A simple exercise shows that any map of $E$ to $T$ that agrees with $i d \times\{0\}$ on $\partial E$ is homotopic to the composition of $\phi_{k}$, for some $k$, and some power of a Dehn twist about $E$. Since $g$ is homotopic to $i d \times\{0\}$ within $X$, the Dehn twist is un-necessary, so $g$ is homotopic to $f_{k}$ in $(\hat{X}, \partial G \times[-1,1])$.

We recall that we will be considering the case where $\left\{\rho_{n}\right\} \subset A H(S)$ converges to $\rho,\left\{\rho_{n}\left(\pi_{1}(S)\right)\right\}$ converges to $\hat{\Gamma}$, and there is a level surface $F \subset N_{\rho}^{0}$ and a collection $E$ of incompressible annuli in $F$ so that the covering map $\pi: N_{\rho}^{0} \rightarrow \hat{N}^{0}$ is an embedding on $F-E$ and (non-trivially) wraps each component of $E$ around a toroidal component of $\partial \hat{N}_{\rho}^{0}$. We also have, for large enough $n$, a 2-bilipschitz map $\psi_{n}: \hat{N} \rightarrow N_{\rho_{n}}$ defined on a regular neighborhood of $\pi(F)$ so that each component of $\psi_{n}(\pi(E))$ bounds a Margulis tube in $N_{\rho_{n}}$. The following lemma gives information about the image of a meridian of a component of $\pi(E)$

Lemma 3.2. Let $G$ be a compact surface, $e \in \mathscr{C}(G)$ and let $\hat{Z}$ be the spine for $\hat{X}$ constructed above. Suppose that $\psi: \hat{Z} \rightarrow M$ is an embedding into a 3-manifold $M$ such that $\psi(T)$ bounds a solid torus $U$ disjoint from $\psi(\hat{Z})$, and $\psi(l)$ is homotopic to the core curve of $U$.

Then there exists $s \in \mathbb{Z}$ such that

(1) $\psi(m+s l)$ bounds a disk in $U$, 
(2) $\psi \circ f_{0}: G \rightarrow M$ is homotopic to $\psi \circ f_{k} \circ D^{k s}$ for all $k$, and

(3) $\psi \circ f_{1}$ is homotopic to $\psi \circ f_{k} \circ D^{(k-1) s}$ for all $k$

where $D: G \rightarrow G$ is a right Dehn twist about $E$.

Proof. Since $\psi(l)$ is homotopic to the core curve of $U$, it is a longitude for $U$. So, the meridian $m_{U}$ for $U$ will intersect $\psi(l)$ exactly once. Therefore, the pre-image $\psi^{-1}\left(m_{U}\right)$ of the meridian will intersect $l$ exactly once and must be of the form $m+s l$ for some $s \in \mathbb{Z}$.

If $s=0$ then (2) and (3) hold, since we may extend $\psi$ to an embedding $\bar{\psi}: X \rightarrow$ $M$ and $f_{0}$ is homotopic to $f_{k}$ within $X$ for all $k$.

We now define a map $h: \hat{Z} \rightarrow \hat{Z}$ which allows us to reduce to the $s=0$ case. Let $h$ be the identity on $\hat{Z}-A$ and let $\left.h\right|_{A}=D_{A}^{-s}$ where $D_{A}$ is the right Dehn twist about the core curve of $A$ so that $h_{*}(m)=m+s l$. Then $\psi \circ h: \hat{Z} \rightarrow M$ is an embedding so that $\psi \circ h(T)$ bounds $U$ and $\psi \circ h(m)$ bounds a disk in $U$. Therefore, for any $k$, $\psi \circ h \circ f_{0}$ is homotopic to $\psi \circ h \circ f_{k}$. The $f_{k}$-pre-image of $A$ in $G$ is a collection of $k$ parallel annuli and the map $h \circ f_{k}$ is equal to pre-composing $f_{k}$ with $s$ Dehn twists in each of the $k$ annuli. As $s$ Dehn twists in $k$ parallel annuli is homotopic to $k s$ Dehn twists in a single annulus we have that $h \circ f_{k}: G \rightarrow Z$ is homotopic to $f_{k} \circ D^{k s}$ for all $k$. Properties (2) and (3) follow immediately.

3.2. Wrapping multicurves and wrapping numbers. In this section, we analyze how compact cores for algebraic limits immerse into geometric limits. We identify the wrapping multicurve and produce a level surface in the algebraic limit whose projection to the geometric limit is embedded off of a collar neighborhood of the wrapping multicurve. At the end of the section, we define the wrapping numbers of the wrapping multicurves.

Proposition 3.3. Suppose that $\left\{\rho_{n}\right\} \subset A H(S), \lim \rho_{n}=\rho$, and $\left\{\rho_{n}\left(\pi_{1}(S)\right)\right\}$ converges geometrically to $\hat{\Gamma}$. Let $\hat{N}=\mathbb{H}^{3} / \hat{\Gamma}$ and let $\pi: N_{\rho} \rightarrow \hat{N}$ be the obvious covering map. There exists a level surface $F$ in $N_{\rho}$, a multicurve $q=\left\{q_{1}, \ldots, q_{r}\right\}$ on $F$, and an open collar neighborhood $Q=\operatorname{collar}(q) \subset F$, so that

(1) $\pi$ restricts to an embedding on $F-Q$.

(2) $l_{\rho}(q)=0$ and if $Q_{i}$ is the component of $Q$ containing $q_{i}$, then $\left.\pi\right|_{Q_{i}}$ is an immersion, which is not an embedding, into the boundary $T_{i}$ of a cusp region $V_{i}$.

(3) If $\hat{J}$ is a (closed) regular neighborhood of $\pi(F)$ in $\hat{N}^{0}$, then $\hat{J}$ is homeomorphic to $F \times[-1,1] \backslash\left(Q \times\left(-\frac{1}{2}, \frac{1}{2}\right)\right)$ and $\partial_{1} J=\partial \hat{J}-\pi\left(\partial N_{\rho}^{0}\right)$ is incompressible in $\hat{N}^{0}$. In particular, $\pi_{1}(\hat{J})$ injects into $\hat{\Gamma}$.

(4) If $d$ is a downward-pointing parabolic in $N_{\rho}$, then $i(d, q)=0$. Moreover, if $d$ is not a component of $q$ and $c$ is a curve in $S$ which intersects $d$, then the geodesic representative $c^{*}$ lies above $d^{*}$ in $N_{\rho_{n}}$ for all large enough $n$.

Analogously, if $d$ is an upward-pointing parabolic in $N_{\rho}$, then $i(d, q)=0$. Moreover, if $d$ is not a component of $q$ and $c$ is a curve in $S$ which intersects $d$, then the geodesic representative $c^{*}$ lies below $d^{*}$ in $N_{\rho_{n}}$ for all large enough $n$. 
(5) If there is a compact core for $N_{\rho}$ which embeds, under $\pi$, in $\hat{N}$, then $q$ is empty.

We will call $q$ the wrapping multicurve of the triple $\left(\left\{\rho_{n}\right\}, \rho, \hat{\Gamma}\right)$. We say that a parabolic curve $d$ for $\rho$ is an unwrapped parabolic for the triple $\left(\left\{\rho_{n}\right\}, \rho, \hat{\Gamma}\right)$ if it does not lie in the wrapping multicurve $q$.

Proof. Let $\widehat{\mathscr{H}}$ be an invariant collection of horoballs for the parabolic elements of $\hat{\Gamma}$ and let $\mathscr{H}$ be the subset of $\widehat{\mathscr{H}}$ consisting of horoballs based at fixed points of parabolic elements of $\rho\left(\pi_{1}(S)\right)$. Let

$$
\hat{N}_{\rho}^{0}=\left(\mathbf{H}^{3}-\bigcup_{H \in \widehat{\mathscr{H}}} H\right) / \hat{\Gamma} \text { and } N_{\rho}^{0}=\left(\mathbf{H}^{3}-\bigcup_{H \in \mathscr{H}} H\right) / \rho(\Gamma)
$$

and let $(M, P)$ be a relative compact core for $N_{\rho}^{0}$.

Let $A$ be a maximal collection of disjoint, nonparallel essential annuli in $(M, P)$ with one boundary component in $P$. Since one may identify $M$ with $S \times[-1,1]$ so that $\partial S \times[-1,1]$ is identified with a collection of components of $P$, one may identify $A$ with $a \times[-1,1]$ where $a=\left\{q_{1}, \ldots, q_{t}\right\}$ is a disjoint collection of simple closed curves on $S$. Let $R$ be the complement in $S$ of a collar neighborhood of the multicurve $a$. Let $\left\{R_{j}\right\}$ be the components of $R$ and let $\Gamma^{j}=\rho\left(\pi_{1}\left(R_{j}\right)\right)$. Notice that an element of $\Gamma^{j}$ is parabolic if and only if it is conjugate to an element of $\rho\left(\pi_{1}\left(\partial R_{j}\right)\right)$. Proposition 6.4 in [12] implies that there exists a proper embedding $h: R_{j} \rightarrow \hat{N}^{0}$ such that $h_{*}\left(\pi_{1}\left(R_{j}\right)\right)$ is conjugate to $\Gamma^{j}$ for each $j$. In particular, $h\left(\partial R_{j}\right) \subset \partial \hat{N}^{0}$.

We now construct $F$. For each $j$, let $F_{j}$ be a lift of $h\left(R_{j}\right)$ to $N_{\rho}$. For each $i$, let $Q_{i}$ be the annulus in $\partial N_{\rho}^{0}$ joining two component of $\bigcup \partial F_{j}$ whose core curve is homotopic to $q_{i}$. Then $F=\bigcup F_{j} \cup \bigcup Q_{i}$ is a level surface for $N_{\rho}^{0}$.

We re-order $\left\{q_{1}, \ldots, q_{r}, q_{r+1}, \ldots, q_{t}\right\}$ so that if $i \leq r$, then $\left.\pi\right|_{Q_{i}}$ is not an embedding, while if $i>r$, then $\left.\pi\right|_{Q_{i}}$ is an embedding. Let $q=\left\{q_{1}, \ldots, q_{r}\right\}$ and $Q=Q_{1} \cup \cdots \cup Q_{r}$. Conditions (1) and (2) are satisfied by construction.

Let $\hat{J}$ be a (closed) regular neighborhood of $\pi(F)$ in $\hat{N}^{0}$. By construction, $\hat{J}$ is homeomorphic to $F \times[-1,1] \backslash\left(Q \times\left(-\frac{1}{2}, \frac{1}{2}\right)\right)$. We first prove that

$$
\partial_{1} \hat{J}=\partial \hat{J}-\pi\left(\partial \hat{N}_{\rho}^{1}\right) \cong F \times\{-1,1\}
$$

is incompressible in $\hat{N}^{0}$. Since $\partial_{1} \hat{J}$ is clearly incompressible in $\hat{J}$, we only need to check that $\partial_{1} \hat{J}$ is incompressible in $\hat{N}^{0}-\operatorname{int}(\hat{J})$. Each component $E$ of $\partial_{1} \hat{J}$ is homeomorphic to $S$. If $E$ is not incompressible in $\hat{N}^{0}-\hat{J}$, then there exists an embedded disk $D$ in $\hat{N}^{0}-\operatorname{int}(\hat{J})$ which is bounded by a homotopically non-trivial curve in $\partial_{1} \hat{J}$.

By Lemma 2.12, there exists, for all large enough $n, Z_{n}$ and a 2-bilipschitz embedding $\psi_{n}: Z_{n} \rightarrow N_{\rho_{n}}$ so that $\hat{J} \cup D \subset Z_{n}$ and if $T$ is a toroidal boundary component of $\hat{J}$, then $\psi_{n}(T)$ bounds a Margulis tube in $N_{\rho_{n}}$. Moreover, if $c$ is a curve in $R_{j} \cap T$, for some $j$, then $\psi_{n}(c)$ is homotopic to the core curve of the Margulis tube. Let $J_{n}$ be the union of $\psi_{n}(\hat{J})$ and all the Margulis tubes bounded by toroidal components 
of $\psi_{n}(\partial \hat{J})$. Then, $J_{n}$ is homeomorphic to $F \times[0,1]$ and $F_{n}=\psi_{n}(\pi(F))$ is homotopic, within $J_{n}$, to a level surface of $J_{n}$. Moreover, Lemma 2.12(4) implies that each level surface of $J_{n}$ is properly homotopic to a level surface in $N_{\rho_{n}}^{1}$ for all large enough $n$. Hence, $\psi_{n}(D)$ is a disk in $N_{\rho_{n}}$ bounded by a homotopically non-trivial curve in an embedded incompressible surface, which is impossible. Therefore, $\partial_{1} \hat{J}$ is incompressible in $\hat{N}^{0}$. Since $\partial \hat{N}^{0}$ is incompressible in $\hat{N}$, it follows that $\pi_{1}(\hat{J})$ injects into $\hat{\Gamma}$. We have established property (3).

We now turn to the proof of property (4). Let $d$ be a parabolic curve for $N_{\rho}$. If $i(d, q) \neq 0$, then $\pi(d)$ is non-peripheral in the regular neighborhood $\hat{J}$ of $\pi(F)$. Since, $\partial_{1} \hat{J}$ is incompressible in $\hat{N}^{0}$, it follows that $\pi(d)$ is non-peripheral in $\hat{N}^{0}$. However, since $\pi(d)$ is associated to a parabolic element of $\hat{\Gamma}$, this is impossible. Therefore, $i(d, q)=0$.

Now suppose that $d$ is an unwrapped downward-pointing parabolic. It remains to show that if $c$ is a curve on $S$ which intersects $d$, then the geodesic representative of $c$ lies above the geodesic representative $d_{n}^{*}$ of $d$ in $N_{\rho_{n}}$ for all sufficiently large $n$.

We first observe that there exists an immersed annulus $A$ in $\hat{N}^{0}$ joining $\pi(d)$ to an essential curve $a$ in the cusp region $V(\pi(d))$ associated to $\pi(d)$ in $\hat{N}$ whose interior is disjoint from $\pi(F)$. We may assume that $\hat{J}$ is disjoint from $V(\pi(d))$. Since $\pi(d)$ is homotopic into $V(d)$, there exists an essential curve $a$ in $\partial V(d)$ which is homotopic to $\pi(d)$. Let $A$ be an immersed annulus in $\hat{N}^{0}$ joining $\pi(d)$ to $a$. If $A$ cannot be chosen so that its interior is disjoint from $\pi(F)$, then there exists a curve $\pi(b)$ in $\pi(F-Q)$ which is homotopic to $\pi(d)$ in $\hat{N}$, but $b$ is not homotopic to $d$ in $F$. Then there exists $\gamma \in \hat{\Gamma}-\rho\left(\pi_{1}(S)\right)$ such that $\gamma \rho(b) \gamma^{-1}=\rho(d)$, so $\rho(b)$ is also parabolic. Let $V(b)$ and $V(d)$ be the distinct cusp regions associated to $b$ and $d$ in $N_{\rho}$. Since $\pi(b)$ is homotopic to $\pi(d), \pi(V(b))=\pi(V(d))$. Lemma 2.12 implies that $\rho_{n}(b)$ is homotopic to $\rho_{n}(d)$ in $N_{\rho_{n}}$ for all large enough $n$, which is a contradiction. Therefore, we may assume that the interior of $A$ is disjoint from $\pi(F)$ as claimed.

We next observe that $F_{n}=\psi_{n}(\pi(F))$ lies above $d_{n}^{*}$ for all large enough $n$. The annulus $A$ lifts to an annulus in $N_{\rho}$ which lies below $F$. We may assume that, for all large enough $n, A \subset Z_{n}$ and $\psi_{n}(a)$ is an essential curve in the boundary of the Margulis tube associated to $d_{n}^{*}$ in $N_{\rho_{n}}$. Let $R$ be the component of $F-Q$ containing $d$. Since $\ell_{\rho}(\partial R)=0$, a result of Otal [35, Theorem A] implies that, for all large enough $n$, the geodesic representative of each component of $\partial R$ is unknotted in $N_{\rho_{n}}$. Lemma 2.9 in [11] then implies that $\psi_{n}(\pi(R))$ is a level subsurface of $N_{\rho_{n}}^{1}$ for all large enough $n$. Since $\psi_{n}(A)$ lies below $\psi_{n}(\pi(R)), d_{n}^{*}$ lies below the embedded subsurface $\psi_{n}(\pi(R))$. Lemma 2.7 in [11] then implies that $d_{n}^{*}$ also lies below $F_{n}$.

If $c$ is a curve on $S$ which intersects $d$ essentially, then $c$ has a representative $c_{n}$ on $F_{n}$ of length at most $L(c)$, for all $n$, so there exists a homotopy from $c_{n}$ to either $c_{n}^{*}$ or to a Margulis region in $N_{\rho_{n}}$ associated to $c$ which has tracks of length at most $D(c)$, where $D(c)$ depends only on $L(c)$ (see [12, Lemma 2.6]). If $d\left(\partial \mathbb{T}_{\varepsilon}^{n}, d_{n}^{*}\right)>D(c)$, then this homotopy will miss $d_{n}^{*}$, which implies that $c_{n}^{*}$ lies above $d_{n}^{*}$. However, this will be the case if $\ell_{\rho_{n}}(d)$ is sufficiently close to 0 , which occurs for all large enough $n$. 
The proof of property (4) for unwrapped upward-pointing parabolics is analogous.

If $q$ is non-empty and there is a compact core for $N$ which embeds in $N_{\rho}$, then $\left.\pi\right|_{F}$ is homotopic to an embedding. However, since $\partial_{1} \hat{J}$ has incompressible boundary, this implies that $\left.\pi\right|_{F}$ is homotopic to an embedding within $\hat{J}$, which is clearly impossible. This establishes (5) and completes the proof.

Let $q_{i}$ be a curve of $q$. We will now define the wrapping number of $q_{i}$ with respect to $\left(\left\{\rho_{n}\right\}, \rho, \Gamma\right)$. Consider the manifolds $X$ and $\hat{X}$ defined in section 3.1 with $G=F$ and $e=q_{i}$. From (3) we get an inclusion $\imath: \hat{J} \rightarrow \hat{X}$. Furthermore, $\imath \circ \pi: F \rightarrow \hat{X}$ is homotopic, as a map into $X$, to $i d \times\{0\}$. Lemma 3.1 implies that there is a unique $k \in \mathbb{Z}$ such that $\imath \circ \pi$ is homotopic to $f_{k}$ as maps into $\hat{X}$. We then define the wrapping numbers $w^{+}\left(q_{i}\right)=k$ and $w^{-}\left(q_{i}\right)=k-1$. Of course, it is clear that $w^{+}$determines $w^{-}$, but as we will see, it is convenient to keep track of both numbers. Notice that the parabolic corresponding to a curve $q_{i}$ is downward pointing (in $N_{\rho}$ ) if and only if $w^{+}\left(q_{i}\right)>0$.

If $q=\left\{q_{1}, \ldots, q_{r}\right\}$, then we get $r$-tuples

$$
w^{+}(q)=\left(w^{+}\left(q_{1}\right), \ldots, w^{+}\left(q_{r}\right)\right) \text { and } w^{-}(q)=\left(w^{-}\left(q_{1}\right), \ldots, w^{-}\left(q_{r}\right)\right) .
$$

Remarks: (1) Our wrapping numbers are closely related to the wrapping coefficients discussed in Brock-Canary-Minsky [12, Section 3.6] and the wrapping numbers defined by Evans-Holt [17].

(2) Lemma 3.1 may be viewed as a special case (and amplification) of the analysis carried out in section 4 of Anderson-Canary-McCullough [3]. In the language of that paper, the subsurfaces $\left\{F_{j}\right\}$ are the relative compact carriers of the precisely embedded system $\left\{\rho\left(\pi_{1}\left(R_{j}\right)\right\}\right.$ of generalized web subgroups.

\section{ASYMPTOTIC BEHAVIOR OF END INVARIANTS IN CONVERGENT SEQUENCES}

In this section, we prove that if a sequence of Kleinian surface groups converges, then some subsequence of the end invariants bounds projections. Along the way we will see that the sequence of end invariants also predicts the parabolics in the algebraic limit and whether they are upward-pointing or downward-pointing. In combination with results from [11] we see that the asymptotic behavior of the end invariants predicts all the lamination and curve components of the end invariants of the algebraic limit. Predicting the conformal structures which arise is significantly more mysterious. We also see that the asymptotic behavior of the end invariants predicts the wrapping multicurve and the associated wrapping numbers in any geometric limit.

Theorem 4.1. Suppose that $\left\{\rho_{n}\right\}$ is a convergent sequence in $A H(S)$ with end invariants $\left\{v_{n}^{ \pm}\right\}$and that $\lim \rho_{n}=\rho$. Then there exists a subsequence $\left\{\rho_{j}\right\}$ such that the sequence $\left\{v_{j}^{ \pm}\right\}$bounds projections.

Furthermore, if $\left\{\rho_{j}\right\}$ is a subsequence such that $\left\{v_{j}^{ \pm}\right\}$bounds projections, then 
(1) $\ell_{\rho}(d)=0$ if and only if $d$ is a combinatorial parabolic for the sequence $\left\{v_{j}^{ \pm}\right\}$

(2) A parabolic curve d is upward-pointing in $N_{\rho}$ if and only if

$$
\left|m\left(v_{j}^{+}, d, \mu\right)\right|-\left|m\left(v_{j}^{-}, d, \mu\right)\right| \rightarrow+\infty .
$$

(3) A lamination $\lambda \in \mathscr{E} \mathscr{L}(Y)$ is an ending lamination for an upward-pointing (respectively downward-pointing) geometrically infinite end for $N_{\rho}$ if and only if $\left\{\pi_{Y}\left(v_{j}^{+}\right)\right\}$(respectively $\left\{\pi_{Y}\left(v_{j}^{-}\right)\right\}$) converges to $\lambda \in \mathscr{C}(Y) \cup \mathscr{E} \mathscr{L}(Y)$.

(4) If $\left\{\rho_{j}\left(\pi_{1}(S)\right)\right\}$ converges geometrically to $\hat{\Gamma}$, then the wrapping multicurve for $\left(\left\{\rho_{j}\right\}, \rho, \Gamma\right)$ is the collection of combinatorial wrapping parabolics given by $\left\{v_{j}^{ \pm}\right\}$and if is a wrapping parabolic, then the combinatorial wrapping number $w(d)$ agree with the actual wrapping number $w^{+}(d)$.

Remark: In general, it is necessary to pass to a subsequence since the phenomenon of self-bumping (see McMullen [29] or Bromberg-Holt [13]) assures that you can have a convergent sequence with one subsequence where the wrapping multicurve is empty and another subsequence where the wrapping multicurve is non-empty.

Proof. We first prove that any geodesic joining $v_{n}^{+}$to $v_{n}^{-}$always intersects some bounded set.

Lemma 4.2. Suppose that $\left\{\rho_{n}\right\}$ is a sequence in $A H(S)$ converging to $\rho$. Let $v_{n}^{ \pm}$ be the end invariants of $\rho_{n}$. There is a bounded set $\mathscr{B} \subset \mathscr{C}(S)$ such that every geodesic joining $\pi_{S}\left(v_{n}^{+}\right)$to $\pi_{S}\left(v_{n}^{-}\right)$intersects $\mathscr{B}$.

Proof. If $a$ is any curve in $\mathscr{C}(S)$, then there is a uniform upper bound $L_{a}$ on the length $l_{\rho_{n}}(a)$ for all $n$. It follows from Theorem 2.6 that $a$ lies within $D\left(L_{a}\right)=D_{0}\left(\max \left\{L_{a}, L_{0}\right\}\right)$ of any geodesic joining $\pi_{S}\left(v_{n}^{+}\right)$to $\pi_{S}\left(v_{n}^{-}\right)$. One may thus choose $\mathscr{B}$ to be a neighborhood of $a$ in $\mathscr{C}(S)$ of radius $D\left(L_{a}\right)$.

We next show that $\ell_{\rho}(d)$ is non-zero if and only if $\left\{m\left(v_{n}^{+}, \mu, d\right)\right\}$ and $\left\{m\left(v_{n}^{-}, \mu, d\right)\right\}$ are both eventually bounded for any marking $\mu$. This is a fairly immediate consequence of work of Minsky, namely Theorem 2.5 and the Short Curve Theorem of [31].

Lemma 4.3. Suppose that $\left\{\rho_{n}\right\}$ is a sequence in $A H(S)$ converging to $\rho$. Let $v_{n}^{ \pm}$ be the end invariants of $\rho_{n}$ and let $\mu$ be a marking on $S$. Then, $l_{\rho}(d)>0$ if and only if $\left\{m\left(v_{n}^{+}, d, \mu\right)\right\}$ and $\left\{m\left(v_{n}^{-}, d, \mu\right)\right\}$ are both eventually bounded.

Proof. First suppose that $\left\{m\left(v_{n}^{\beta}, d, \mu\right)\right\}$ is not eventually bounded, then either $\left\{\frac{1}{l_{v_{n}^{\beta}}(d)}\right\}$ is not eventually bounded or $\left\{\sup _{d \subset \partial Y} d_{Y}\left(v_{n}^{\beta}, \mu\right)\right\}$ is not eventually bounded. If $\left\{\frac{1}{l_{v_{n}^{\beta}}(d)}\right\}$ is not eventually bounded, then there exists a subsequence $\left\{\rho_{j}\right\}$ of $\left\{\rho_{n}\right\}$ so that $\left\{\ell_{\rho_{j}}(d)\right\}$ converges to 0 , which implies that $\ell_{\rho}(d)=0$. If $\left\{\sup _{d \subset \partial Y} d_{Y}\left(v_{n}^{\beta}, \mu\right)\right\}$ is not eventually bounded, then either

(1) there exists a subsequence for which $d$ is always a component of $v_{j}^{\beta}$, or

(2) there exists a sequence of subsurfaces $Y_{j}$ such that $d \subset \partial Y_{j}$ and $d_{Y_{j}}\left(v_{j}^{\beta}, \mu\right) \rightarrow \infty$. 
In case $(1), \ell_{\rho_{j}}(d)=0$ for all $j$, so $\ell_{\rho}(d)=0$. In case $(2)$, since $\ell_{\rho_{j}}(\mu)$ is eventually bounded and $\pi_{Y_{j}}\left(\mu_{j}^{\beta}\right) \in \overline{\pi_{Y_{j}}\left(\mathscr{C}\left(\rho, L_{B}\right)\right)}$ where $L_{B}$ is the Bers constant for $S$ (see Brock-Bromberg-Canary-Minsky [11, Section 2]), we see that $\operatorname{diam}\left(\pi_{Y_{j}}(\mathscr{C}(\rho, L)) \rightarrow \infty\right.$ for some $L$. Theorem 2.5 then implies that $\lim \ell_{\rho_{j}}(d)=0$, so again $\ell_{\rho}(d)=0$. Therefore, in all cases, if $\left\{m\left(v_{n}^{\beta}, d, \mu\right)\right\}$ is not eventually bounded, then $\ell_{\rho}(d)=0$. It follows that if $l_{\rho}(d)>0$ then $\left\{m\left(v_{n}^{+}, d, \mu\right)\right\}$ and $\left\{m\left(v_{n}^{-}, d, \mu\right)\right\}$ are both eventually bounded.

If $\ell_{\rho}(d)=0$, then Minsky's Short Curve Theorem [31] implies that at least one of $\left\{\frac{1}{\ell_{v_{n}^{+}}(d)}\right\},\left\{\frac{1}{\ell_{v_{n}^{-}}(d)}\right\}$, and $\left\{\sup _{d \subset \partial Y} d_{Y}\left(v_{n}^{+}, v_{n}^{-}\right)\right\}$is not eventually bounded. (For a similar restatement of the Short Curve Theorem in the quasifuchsian case see Brock-Bromberg-Canary-Minsky [10, Thm. 2.2].) It follows that $\left\{m\left(v_{n}^{\beta}, d, \mu\right)\right\}$ is not eventually bounded for some $\beta \in\{ \pm\}$.

We now pass to a subsequence $\left\{\rho_{j}\right\}$ of $\left\{\rho_{n}\right\}$ so that $\left\{\rho_{j}\left(\pi_{1}(M)\right)\right\}$ converges geometrically to $\hat{\Gamma}$. Let $\hat{N}=\mathbf{H}^{3} / \Gamma$. Let $F, q=\left\{q_{1}, \ldots, q_{s}\right\}$ and $Q$, be the level surface, wrapping multicurve and collar neighborhood of $q$ provided by Proposition 3.3. Let

$$
f: S \rightarrow F
$$

be a homeomorphism such that $f_{*}=\rho \in A H(S)$ and let $\hat{J}$ be a closed regular neighborhood of $\pi(F)$ in $\hat{N}^{0}$.

The following lemma characterizes the asymptotic behavior of the end invariants relative to an unwrapped parabolic.

Lemma 4.4. Suppose that $\left\{\rho_{j}\right\}$ is a sequence in $A H(S)$ converging to $\rho$ such that $\left\{\rho_{j}\left(\pi_{1}(S)\right)\right\}$ converges geometrically to $\hat{\Gamma}$ and that $d$ is an unwrapped parabolic for the triple $\left(\left\{\rho_{j}\right\}, \rho, \hat{\Gamma}\right)$. Then,

(1) if $d$ is a downward-pointing cusp in $N_{\rho}$, then $\left\{m\left(v_{j}^{+}, d, \mu\right)\right\}$ is eventually bounded, and

(2) if $d$ is an upward-pointing cusp in $N_{\rho}$, then $\left\{m\left(v_{j}^{-}, d, \mu\right)\right\}$ is eventually bounded.

Proof. Let $d$ be a downward-pointing cusp in $N_{\rho}$ and let $L_{\mu}$ be an upper bound for the length of $\mu$ in $N_{\rho_{j}}$ for all $j$. Let $a$ be a curve in $\mu$ which crosses $d$. Proposition 3.3 guarantees that $a^{*}$ lies above $d^{*}$ in $N_{\rho_{j}}$ for all sufficiently large $j$. Theorem 2.8 then implies that, for all sufficiently large $j, d_{Y}\left(a, v_{j}^{+}\right)<D\left(L_{\mu}\right)$ if $Y \subset S$ is any subsurface with $d$ in its boundary. Therefore, $\left\{\sup _{d \subset \partial Y} d_{Y}\left(v_{j}^{+}, \mu\right\}\right)$ is eventually bounded.

It remains to check that there is an eventual lower bound on $l_{v_{j}^{+}}(d)$. (For a similar argument in the quasifuchsian case, see [10, Lemma 2.5].) Since $l_{\rho_{j}}(a)<L_{\mu}$ for all $j$, there exists $\varepsilon>0$ so that the geodesic representative $a_{j}^{*}$ of $a$ in $N_{\rho_{j}}$ misses $\mathbb{T}_{\varepsilon}(d)$ for all $j$. The convex core $\partial C\left(N_{\rho_{j}}\right)$ of $N_{\rho_{j}}$ is the smallest convex submanifold of $N_{\rho_{j}}$ containing all the closed geodesics. Epstein, Marden and Markovic [16, Theorem 3.1] proved that there is a 2-Lipschitz map $f_{j}: \partial_{c} N_{\rho_{j}} \rightarrow \partial C\left(N_{\rho_{j}}\right)$ so that $f_{j}$ extends to a strong deformation retraction of $\partial_{c} N_{\rho_{j}} \cup N_{\rho_{j}}$ onto $C\left(N_{\rho_{j}}\right)$. 
In particular, if $R_{j}$ is a downward-pointing component of $\partial_{c} N_{\rho_{j}}$, then no closed geodesic in $N_{\rho_{j}}$ lies below $f\left(R_{j}\right)$. If $l_{v_{j}^{+}}(d)=l_{j}<\varepsilon / 2$, then there is a representative $d_{j}$ of $d$ in the image $f_{j}\left(R_{j}\right)$ of a downward-pointing component $R_{j}$ of $\partial_{c} N_{\rho_{j}}$ which has length at most $2 l_{j}<\varepsilon$, so is contained in $\mathbb{T}_{\varepsilon}\left(d_{j}\right)$. Therefore, $a_{j}^{*}$ cannot intersect $d_{j}$, so is disjoint from $f\left(R_{j}\right)$. It follows that $f\left(R_{j}\right)$ lies below $a_{j}^{*}$, which implies that $d_{j}$ lies below $a_{j}^{*}$. Since $d_{j}$ is homotopic to $d_{j}^{*}$ within $\mathbb{T}_{\varepsilon}\left(d_{j}\right)$ and $a_{j}^{*}$ is disjoint from $\mathbb{T}_{\varepsilon}\left(d_{j}\right)$, we see that $d_{j}^{*}$ lies below $a_{j}^{*}$. However, this contradicts Proposition 3.3 , so $l_{v_{j}^{+}}(d) \geq \varepsilon / 2$ for all sufficiently large $j$ which completes the proof for downward-pointing cusps.

The proof in the case that $d$ is an upward-pointing cusp is similar.

The situation is more complicated for wrapped parabolics.

We will abuse notation by letting $q$ also denote the multicurve $f^{-1}(q) \subset S$ and by letting $Q$ denote the subsurface $f^{-1}(Q)$ of $S$. Let $X=S \times[-1,1]$ and $\hat{X}=X-V$ where $V=Q \times\left(-\frac{1}{2}, \frac{1}{2}\right) \subset X$ is a union of open solid tori in the homotopy class of $q$. Set

$$
\hat{Z}=(S-Q) \times\{0\} \cup \partial V \subset \hat{X} .
$$

If $q$ is a single curve, then we are in the situation of section 3.1 with $G=S$ and $e=q$. We encourage the reader to focus on this situation when first reading the section.

In general, if $q=\left\{q_{1}, \ldots, q_{r}\right\}$, we divide $S$ into a collection of overlapping subsurfaces $\left\{G_{1}, \ldots, G_{r}\right\}$ defined as follows: $G_{i}$ is the connected component of $S-\left(Q-Q_{i}\right)$ that contains $Q_{i}$. One may then divide $X$ up into overlapping submanifolds $\left\{X_{1}, \ldots, X_{r}\right\}$ where $X_{i}=G_{i} \times[-1,1]$. Similarly, one may divide $\hat{X}$ up into submanifolds $\left\{\hat{X}_{1}, \ldots, \hat{X}_{r}\right\}$ with $\hat{X}_{i}=X_{i}-V_{i}$ where $V_{i}=Q_{i} \times\left(-\frac{1}{2}, \frac{1}{2}\right) \subset X_{i}$. Let $T_{i}$ be the toroidal boundary component of $\hat{X}_{i}$.

Proposition 3.3 implies that we may identify $\hat{J}$ with $\hat{X}$. If $k=\left(k_{1}, \ldots, k_{r}\right)$ we may define a map $f_{k}: S \rightarrow \hat{J}$ which agrees on each $G_{i}$ with the map $f_{k_{i}}: G_{i} \rightarrow \hat{X}_{i}$ defined in section 3.1. Each of the $f_{k}$ determines a representation $\left(f_{k}\right)_{*}$ in $A H(S)$. Since $f$ is homotopic to $f_{w^{+}(q)}$, we see that $\left(f_{w^{+}(q)}\right)_{*}=\rho$.

Given a component $Q_{i}$ of $Q$ we denote by $D_{i}: S \rightarrow S$ the right Dehn twist about $Q_{i}$. For an $r$-tuple $k=\left(k_{1}, \ldots, k_{r}\right)$, we set $D_{q}^{k}=D_{1}^{k_{1}} \circ \ldots \circ D_{r}^{k_{r}}$.

Lemma 4.5. For all large enough $j$, there exists a $r$-tuple $s_{j}=\left(s_{1, j}, \ldots, s_{r, j}\right)$ such that $\rho_{j}^{+}=\rho_{j} \circ\left(D_{q}^{w^{+}(q) s_{j}}\right)_{*}$ and $\rho_{j}^{-}=\rho_{j} \circ\left(D_{q}^{w^{-}(q) s_{j}}\right)_{*}$ have the following properties:

(1) the sequences $\left\{\rho_{j}^{+}\right\}$and $\left\{\rho_{j}^{-}\right\}$converge in $A H(S)$ to $\rho^{+}$and $\rho^{-}$.

(2) If $q_{i}$ is a component of $q$, then $q_{i}$ is an upward pointing parabolic in $N_{\rho^{-}}$ and a downward pointing pararabolic in $N_{\rho^{+}}$and is unwrapped in the triples $\left(\left\{\rho_{j}^{+}\right\}, \rho^{+}, \hat{\Gamma}\right)$ and $\left(\left\{\rho_{j}^{-}\right\}, \rho^{-}, \hat{\Gamma}\right)$.

(3) For each $i, \lim \left|s_{i, j}\right|=+\infty$.

Proof. For all large enough $j$, there exists a 2-bilipschitz embedding $\psi_{j}: \hat{J} \rightarrow N_{\rho_{j}}$ such that each component of $\psi_{j} \circ \phi(\partial V)$ bounds a Margulis tube in $N_{\rho_{j}}$ and $\psi_{j}(\hat{J})$ is disjoint from the interior of these tubes. Moreover, $\left(\psi_{j} \circ \hat{f}\right)_{*}$ is conjugate to 
$\rho_{j}$. In particular, if $l$ is the longitude of any component $T$ of $\partial V$, then $\psi_{j}(l)$ is a longitude of the Margulis tube bounded by $\psi_{j}(T)$.

Given $j \in \mathbb{N}$, Lemma 3.2 applied to $G=G_{i}$ and $e=q_{i}$ implies that for all $i$, there exists $s_{i, j}$ so that if $m_{i}$ and $l_{i}$ are the meridian and longitude of $T_{i}$, then $\psi_{j}\left(m_{i}+s_{i, j} l_{i}\right)$ bounds a meridian of $\psi_{j}\left(T_{i}\right)$. We set $f^{-}=f_{(0, \ldots, 0)}$ and $f^{+}=f_{(1, \ldots, 1)}$ and let $\rho^{+}=f_{*}^{+}$and $\rho^{-}=f_{*}^{-}$. Lemma 3.2 implies that $\psi_{j} \circ f^{+}$is homotopic to $f \circ D_{q}^{w^{+}(q) s_{j}}$ and that $\psi_{j} \circ f^{+}$is homotopic to $f \circ D_{q}^{w^{-}(q) s_{j}}$. It follows that $\left\{\rho_{j}^{+}\right\}$ converges to $\rho^{+}$and that $\left\{\rho_{j}^{-}\right\}$converges to $\rho^{-}$. This establishes property (1) and property (2) is true by construction.

It remains to establish property (3). Notice that since $\lim \ell_{\rho_{j}}\left(q_{i}\right)=0$, the diameter of the Margulis tube bounded by $\psi_{j}\left(T_{i}\right)$ is diverging to $+\infty$. It follows that the length of the meridian of $\psi_{j}\left(T_{i}\right)$ diverges to $+\infty$. Since $\psi_{j}$ is 2-bilipschitz, there is a uniform upper bound on the lengths of $\psi_{j}\left(l_{i}\right)$ and $\psi_{j}\left(m_{i}\right)$. Since the meridian of $\psi_{j}\left(T_{i}\right)$ is homotopic to $\psi_{j}\left(m_{i}+s_{i, j} l_{i}\right)$, we must have $\lim \left|s_{i, j}\right|=+\infty$.

We can now easily assemble the proof of Theorem 4.1. We first show that if $\left\{\rho_{n}\right\}$ converges, then there is a subsequence $\left\{\rho_{j}\right\}$ so that $\left\{v_{j}^{ \pm}\right\}$bounds projections. We choose a subsequence so that $\left\{\rho_{j}\left(\pi_{1}(S)\right)\right\}$ converges geometrically. Lemma 4.2 implies that $\left\{v_{j}^{ \pm}\right\}$satisfies condition (a) of the definition of bounding projections. Lemma 4.3 implies that all the curves $d$ which are not parabolic in the algebraic limit satisfy condition (b)(i). Lemma 4.4 implies that if $d$ is an unwrapped parabolic, then it satisfies condition (b)(i), while Lemma 4.5 combined with Lemma 4.4 implies that any wrapped parabolic curve $d$ satisfies condition (b)(ii). Therefore, $\left\{v_{j}^{ \pm}\right\}$bounds projections as claimed.

We now suppose that $\left\{\rho_{j}\right\}$ is a subsequence so that $\left\{\rho_{j}\left(\pi_{1}(S)\right)\right\}$ converges geometrically. Property (1) follows from Lemma 4.3. Property (2) follows from Lemma 4.4 if $d$ is an unwrapped parabolic. Property (2) for wrapped parabolics follows from Lemma 4.4 and the facts, observed in section 3.1, that $w^{-}(q)=$ $w^{+}(q)-1$ and that $d$ is upward pointing if and only if $w^{+}(q)$ is positive. Property (3) comes from Theorem 2.4 ([11, Theorem 1.1]). Property (4) follows from Lemma 4.5.

In general, if $\left\{\rho_{j}\right\}$ is a subsequence of $\left\{\rho_{n}\right\}$ so that $\left\{v_{j}^{ \pm}\right\}$bounds projections. Then every subsequence of $\left\{\rho_{j}\right\}$ has a subsequence $\left\{\rho_{k}\right\}$ so that $\left\{\rho_{k}\left(\pi_{1}(S)\right)\right\}$ converges geometrically. Therefore, every subsequence of $\left\{\rho_{j}\right\}$ has a subsequence for which properties (1)-(4) hold. It is then easily checked that properties (1)-(4) hold for the original sequence $\left\{\rho_{j}\right\}$.

\section{MULTICURVES FROM END INVARIANTS}

In this section, we prove that if the sequence of end invariants bounds projections, then we can find a sequence of pairs of bounded length multicurves which bounds projections.

Proposition 5.1. Suppose that $\left\{\rho_{n}\right\}$ is a sequence in $A H(S)$ with end invariants $\left\{v_{n}^{ \pm}\right\}$. If $\left\{v_{n}^{ \pm}\right\}$bounds projections, then there exists a subsequence $\left\{\rho_{j}\right\}$ and a 
sequence of pairs of multicurves $\left\{c_{j}^{ \pm}\right\}$such that $\left\{\ell_{\rho_{j}}\left(c_{j}^{+} \cup c_{j}^{-}\right)\right\}$is bounded and $\left\{c_{j}^{ \pm}\right\}$bounds projections.

The moral here is quite simple, although unpleasant technical difficulties arise in the actual proof. If $\left\{\rho_{n}\right\}$ is a sequence of quasifuchsian groups, one might hope to be able to choose $c_{n}^{+}$and $c_{n}^{-}$to be minimal length pants decompositions of the top and bottom conformal boundaries of $N_{n}$. There are three technical issues that cause this simple algorithm to fail:

- The $c_{n}^{+}$and $c_{n}^{-}$cannot have curves in common.

- A downward (upward) pointing unwrapped combinatorial parabolic cannot be in $c_{n}^{+}\left(c_{n}^{-}\right)$.

- A wrapped combinatorial parabolic cannot be in either $c_{n}^{+}$or $c_{n}^{-}$.

It is easy to construct examples where the minimal length pants decompositions fail to satisfy any of these technical constraints. To deal with these issues, we will choose $c_{n}^{+}$to be a minimal length pants decomposition of $v_{n}^{+}$which intersects any downward-pointing combinatorial parabolic, any combinatorial wrapped parabolic and any "sufficiently short" curve on $v_{n}^{-}$. We then choose $c_{n}^{-}$to be a minimal length pants decomposition of $v_{n}^{-}$which intersects any curve in $c_{n}^{+}$, any downwardpointing combinatorial parabolic, and any combinatorial wrapped parabolic.

In general, one might hope to choose $c_{n}^{+}$to consist of a minimal length pants decomposition of each geometrically finite subsurface on the "top," a curve for each upward-pointing parabolic and a pants decomposition of each subsurface supporting an upward-pointing geometrically infinite end which is "close enough" to the ending lamination. We will again need to be more careful in the actual proof.

Proof. We first pass to a subsequence, still called $\left\{\rho_{n}\right\}$, so that if $d$ is a curve and $\beta \in\{ \pm\}$, then either $m\left(v_{n}^{\beta}, d, \mu\right) \rightarrow \infty$ or $\left\{m\left(v_{n}^{\beta}, d, \mu\right)\right\}$ is eventually bounded. Let $b^{\beta}$ be the collection of curves such that $m\left(v_{n}^{\beta}, d, \mu\right) \rightarrow \infty$ if and only if $d$ is in $b^{\beta}$. If $d$ lies in $b^{+}$or $b^{-}$, then $d$ is a combinatorial parabolic, while if $d$ lies in both $b^{+}$ and $b^{-}$, then $d$ is a combinatorial wrapped parabolic.

The following lemma implies that $b^{+}$and $b^{-}$are multicurves.

Lemma 5.2. Suppose that $\left\{\rho_{n}\right\}$ is a sequence in $A H(S)$ with end invariants $\left\{v_{n}^{ \pm}\right\}$ and $\left\{v_{n}^{ \pm}\right\}$bounds projections. If $d$ is either an upward-pointing or wrapped combinatorial parabolic and c intersects $d$, then $\left\{m\left(v_{n}^{+}, c, \mu\right)\right\}$ is eventually bounded.

Similarly, if $d$ is either a downward-pointing or wrapped combinatorial parabolic and c intersects $d$, then $\left\{m\left(v_{n}^{-}, c, \mu\right)\right\}$ is eventually bounded.

Proof. We give the proof in the case that $d$ is either an upward-pointing combinatorial parabolic or a combinatorial wrapped parabolic, in which case $m\left(v_{n}^{+}, d, \mu\right) \rightarrow \infty$. The proof of the other case is analogous.

First suppose that $\ell_{v_{n}^{+}}(d) \rightarrow 0$, so $l_{v_{n}^{+}}(c) \rightarrow \infty$ and $d$ is a curve in the base of the (generalized) marking $\mu\left(v_{n}^{+}\right)$(defined in section 2.2) associated to $v_{n}^{+}$for all large enough $n$. In particular, if $c \in \partial Z$, then

$$
d_{Z}\left(\mu, v_{n}^{+}\right) \leq d_{Z}(\mu, d)+d_{Z}\left(d, \mu\left(v_{n}^{+}\right)\right) \leq 2 i(\mu, d)+6 .
$$


(The second inequality follows from Lemma 2.1 and the fact that any two curves in $\mu\left(v_{n}^{+}\right)$intersect at most twice.) Therefore, if $\ell_{v_{n}^{+}}(d) \rightarrow 0$, then $\left\{m\left(v_{n}^{+}, c, \mu\right)\right\}$ is eventually bounded.

Notice that, by reversing the roles of $c$ and $d$ in the previous sentence, we see that if $m\left(v_{n}^{+}, d, \mu\right) \rightarrow \infty$, then $\left\{\ell_{v_{n}^{+}}(c)\right\}$ is bounded away from zero.

So, we may suppose that both $\left\{\ell_{v_{n}^{+}}(d)\right\}$ and $\left\{\ell_{v_{n}^{+}}(c)\right\}$ are bounded away from zero, and that $\sup _{d \subset \partial Y} d_{Y}\left(v_{n}^{+}, \mu\right) \rightarrow \infty$. Therefore, there exists a sequence of subsurfaces $Y_{n}$ with $d \subset \partial Y_{n}$, so that $d_{Y_{n}}\left(v_{n}^{+}, \mu\right) \rightarrow \infty$. It follows that $d_{Y_{n}}\left(v_{n}^{+}, c\right) \rightarrow \infty$. Lemma 2.2 then implies that if $Z$ is a subsurface with $c \in \partial Z$, then

$$
d_{Z}\left(\partial Y_{n}, v_{n}^{+}\right) \leq 4
$$

for all large enough $n$. So,

$$
d_{Z}\left(v_{n}^{+}, \mu\right) \leq d_{Z}\left(\partial Y_{n}, v_{n}^{+}\right)+d_{Z}\left(\partial Y_{n}, \mu\right) \leq 4+d_{Z}(d, \mu)+1
$$

for all large enough $n$. Since $d_{Z}(d, \mu)$ is bounded above by a function of $i(d, \mu)$, $\left\{\sup _{c \subset \partial Z} d_{Z}\left(v_{n}^{+}, \mu\right)\right\}$ is eventually bounded. Therefore, again $\left\{m\left(v_{n}^{+}, c, \mu\right)\right\}$ is eventually bounded.

We next claim that a curve cannot be "short" on both the top and the bottom.

Lemma 5.3. If $\left\{\rho_{n}\right\}$ is a sequence in $A H(S)$ with end invariants $\left\{v_{n}^{ \pm}\right\}$and $\left\{v_{n}^{ \pm}\right\}$ bounds projections, then there exists $\delta_{1}>0$, so that if $d$ is any curve on $S$, then

$$
\max \left\{\ell_{v_{n}^{+}}(d), \ell_{v_{n}^{-}}(d)\right\}>\delta_{1} .
$$

Proof. If not, we may pass to a subsequence so that there exist curves $a_{n}$ so that $\ell_{v_{n}^{+}}\left(a_{n}\right)+\ell_{v_{n}^{-}}\left(a_{n}\right) \rightarrow 0$. Then $a_{n}$ is a curve of $\mu\left(v_{n}^{ \pm}\right)$hence $d_{Y}\left(a_{n}, v_{n}^{ \pm}\right) \leq 5$ for any subsurface $Y$ that intersects $a_{n}$ essentially. If $\left\{a_{n}\right\}$ admits a constant subsequence $a$, then $m^{n a}\left(v_{n}^{+}, a, \mu\right) \rightarrow \infty$ and $m^{n a}\left(v_{n}^{-}, a, \mu\right) \rightarrow \infty$ which is not allowed by condition (b) of the definition of bounding projections. If not, by Lemma 2.3, there is a subsurface $Y$ such that, after taking a subsequence, $d_{Y}\left(\mu, a_{n}\right) \rightarrow \infty$. Then we have $d_{Y}\left(\mu, v_{n}^{ \pm}\right) \rightarrow \infty$ and $d_{Y}\left(v_{n}^{+}, v_{n}^{-}\right) \leq 5$ which contradicts both conditions (b)(i) and (b)(ii). Therefore, no such subsequence can exist and we obtain the desired inequality.

We recall that the Collar Lemma ([14, Theorem 4.4.6]) implies that any two closed geodesics of length at most $2 \sinh ^{-1}(1)$ on any hyperbolic surface cannot intersect. Let $e_{n}^{\beta}$ denote the multicurve on $S$ consisting of curves $d$ such that

$$
\ell_{v_{n}^{\beta}}(d)<\min \left\{2 \sinh ^{-1}(1), \delta_{1}\right\} .
$$

We now describe the construction of $c_{n}^{ \pm}$in the case that $\left\{\rho_{n}\right\}$ is a sequence of quasifuchsian representations, so $v_{n}^{ \pm} \subset \mathscr{T}(S)$ for all $n$. Among the pants decompositions of $S$ which cross every curve in $b^{-} \cup e_{n}^{-}$, choose one, $c_{n}^{+}$, with minimal length in $v_{n}^{+}$. Then among the pants decompositions of $S$ which cross every curve in $b^{+} \cup c_{n}^{+}$choose one, $c_{n}^{-}$, with minimal length in $v_{n}^{-}$. We observe that the resulting sequences have bounded length.

Lemma 5.4. The sequences $\left\{l_{\rho_{n}}\left(c_{n}^{+}\right)\right\}$and $\left\{l_{\rho_{n}}\left(c_{n}^{-}\right)\right\}$are both bounded. 
Proof. Notice that since $\left\{m^{n a}\left(v_{n}^{-\beta}, d, \mu\right)\right\}$ is bounded for all $d \in b^{\beta}$ and $b^{\beta}$ has finitely many components, there exists $\delta_{2}>0$ such that if $d \in b^{\beta}$, then

$$
\ell_{v_{n}^{-\beta}}(d)>\delta_{2} .
$$

Lemma 5.3 implies that if $d$ is a component of $e_{n}^{\beta}$, then $\ell_{v_{n}^{-\beta}}(d) \geq \delta_{1}$.

Therefore, there is a lower bound, $\min \left\{\delta_{2}, \delta_{1}\right\}$, on the length, in $v_{n}^{+}$, of every curve in $b^{-} \cup e_{n}^{-}$. Since $b^{-} \cup e_{n}^{-}$contains a bounded number of curves, it is an easy exercise to check that there is an upper bound on the length of a minimal length pants decomposition of $v_{n}^{+}$intersecting $b^{-} \cup e_{n}^{-}$, hence an upper bound on the length, in $v_{n}^{+}$, of $c_{n}^{+}$.

Since $c_{n}^{+}$crosses every curve in $e_{n}^{-}$, every curve in $c_{n}^{+}$has length, in $v_{n}^{-}$, at least $\min \left\{2 \sinh ^{-1}(1), \delta_{1}\right\}$. Therefore, there is a lower bound, $\min \left\{\delta_{2}, \delta_{1}, 2 \sinh ^{-1}(1)\right\}$, on the length, in $v_{n}^{-}$, of every curve in $c_{n}^{+} \cup b^{+}$. It again follows that there is an upper bound on the length of $c_{n}^{-}$.

Bers [6, Theorem 3] proved that if $d$ is any curve on $S$, then

$$
\ell_{\rho_{n}}(d) \leq 2 \ell_{v_{n}^{\beta}}(d)
$$

for either $\beta=+$ or $\beta=-$. It follows that both $\left\{l_{\rho_{n}}\left(c_{n}^{+}\right)\right\}$and $\left\{l_{\rho_{n}}\left(c_{n}^{-}\right)\right\}$are bounded.

Since $c_{n}^{\beta}$ and the base of the marking $\mu\left(v_{n}^{\beta}\right)$ both have uniformly bounded length in $v_{n}^{\beta}$, there is a uniform upper bound on the intersection number between $c_{n}^{\beta}$ and any base curve of the marking $\mu\left(v_{n}^{\beta}\right)$. Therefore, Lemma 2.1 implies that there exists $K$ so that if $Y \subseteq S$ is not a component of $\operatorname{collar}\left(c_{n}^{\beta}\right)$ or collar $\left(\operatorname{base}\left(v_{n}^{\beta}\right)\right)$, then

$$
d_{Y}\left(c_{n}^{\beta}, v_{n}^{\beta}\right) \leq K .
$$

If $Y$ is a component of $\operatorname{collar}\left(\operatorname{base}\left(v_{n}^{\beta}\right)\right)$ and $c_{n}^{\beta}$ crosses $Y$, then, since $c_{n}^{\beta}$ has bounded length, there is a lower bound on the length of the core curve of $Y$ and hence an upper bound on the length of the transversal to $Y$ in the marking $\mu\left(v_{n}^{\beta}\right)$. Again, this implies an upper bound on the intersection number between the transversal and $c_{n}^{\beta}$, so inequality (5.1) still holds.

Finally, we pass to a subsequence so that, for each $\beta$, if $d$ is any curve then $d$ either lies in $c_{n}^{\beta}$ for all $n$ or for only finitely many $n$. Since $c_{n}^{\beta}$ is a pants decomposition and $c_{n}^{-}$crosses every curve in $c_{n}^{+}$, then for any curve $d$ there exists $\beta(d) \in\{ \pm\}$ and $N(d) \in \mathbb{Z}$ such such that $c_{n}^{\beta}$ crosses $d$ for all $n \geq N(d)$.

The next lemma shows that the properties we have established suffice to show that $\left\{c_{n}^{ \pm}\right\}$bounds projections. We give the statement and the proof in the general case (i.e. $\rho_{n}$ is not assumed to be quasifuchsian).

Lemma 5.5. Let $\left\{v_{n}^{ \pm}\right\}$be a sequence of pairs of end invariants which bounds projections and let $\left\{c_{n}^{ \pm}\right\}$be a sequence of pairs of multicurves on $S$ such that

(1) there exists $K^{\prime}>0$ such that $d_{S}\left(c_{n}^{\beta}, v_{n}^{\beta}\right) \leq K^{\prime}$, 
(2) there exists $K>0$ such that if $d \in \mathscr{C}(S)$, then there exists $M(d) \in \mathbb{N}$ such that if $Y \subset S$ with $d \subset \partial Y, c_{n}^{\beta}$ crosses $d$, then

$$
d_{Y}\left(v_{n}^{\beta}, c_{n}^{\beta}\right) \leq K
$$

for any $\beta \in\{ \pm\}$ and any $n \geq M(d)$,

(3) if $d$ is a wrapped combinatorial parabolic, then $c_{n}^{\beta}$ intersects $d$ for any $\beta \in\{ \pm\}$

(4) if $d$ is an unwrapped downward (respectivley upward) pointing combinatorial parabolic, then $c_{n}^{+}$(resp. $c_{n}^{-}$) intersects $d$, and

(5) if $d$ is not a combinatorial parabolic, then there exists $\beta(d) \in\{ \pm\}$ and $N(d) \in \mathbb{N}$ such that $c_{n}^{\beta(d)}$ crosses $d$ for all $n \geq N(d)$.

Then $\left\{c_{n}^{ \pm}\right\}$bounds projections.

Proof. Since $\left\{v_{n}^{ \pm}\right\}$bounds projection, there exists a bounded set $\mathscr{B}$ so that any geodesic joining $\pi_{S}\left(v_{n}^{+}\right)$to $\pi_{S}\left(v_{n}^{-}\right)$intersects $\mathscr{B}$. By property $(1), d_{S}\left(c_{n}^{\beta}, v_{n}^{\beta}\right)$ is uniformly bounded, so the hyperbolicity of the curve complex implies that any geodesic joining $c_{n}^{+}$to $c_{n}^{-}$lies a bounded Hausdorff distance from a geodesic joining $\pi_{S}\left(v_{n}^{+}\right)$to $\pi_{S}\left(v_{n}^{-}\right)$, and hence lies a bounded distance from $\mathscr{B}$. Therefore, any geodesic joining $c_{n}^{+}$to $c_{n}^{-}$intersects some bounded set $\mathscr{B}^{\prime}$, so $\left\{c_{n}^{ \pm}\right\}$satisfies condition (a) in the definition of bounding projections.

If $d$ is a combinatorial wrapped parabolic, then $d$ crosses both $c_{n}^{+}$and $c_{n}^{-}$(by property (3)), so inequality (5.2) implies that $d$ is a combinatorial wrapped parabolic for $\left\{c_{n}^{ \pm}\right\}$.

If $d$ is an unwrapped combinatorial parabolic, then there exists $\beta=\beta(d)$ so that $d \in b^{-\beta}$, so $\left\{m\left(v_{n}^{\beta(d)}, d, \mu\right)\right\}$ is eventually bounded and $d$ crosses $c_{n}^{\beta(d)}$ for all $n$ (by property (4).) Inequality (5.2) implies that $\left\{m\left(c_{n}^{\beta(d)}, d, \mu\right)\right\}$ is eventually bounded, so $d$ satisfies condition (b)(i).

If $d$ is not a combinatorial parabolic, then there exists $\beta=\beta(d)$ and $N(d)$ such that $d$ crosses $c_{n}^{\beta}$ for all $n \geq N(d)$ (by property (5)). Then, since $\left\{m\left(v_{n}^{\beta}, d, \mu\right)\right\}$ is eventually bounded, inequality (5.2) implies that $\left\{m\left(c_{n}^{\beta}, d, \mu\right)\right\}$ is eventually bounded, so $d$ satisfies condition (b)(i). This completes the proof that condition (b) holds for every curve.

In the quasifuchsian case, Lemma 5.4, inequality (5.1) and Lemma 5.5 imply that $\left\{c_{n}^{ \pm}\right\}$bounds projections, so we have completed the proof of Proposition 5.1 in the quasifuchsian case.

We next suppose that there exists a subsequence $\left\{\rho_{n}\right\}$ such that for all $n$, neither $v_{n}^{+}$or $v_{n}^{-}$is a lamination supported on all of $S$. We list all the simple closed curves on $S$ by fixing a bijection $\alpha: \mathscr{C}(S) \rightarrow \mathbb{N}$.

When choosing the $c_{n}^{+}$on a subsurface $W$ that supports a conformal structure in $v_{n}^{-}$, we will use a procedure similar to the one used in the quasifuchsian case. If $W$ supports a lamination $\lambda$ in $v_{n}^{+}$, we choose a pants decomposition that has bounded length and is "close" to $\lambda$, where close is taken to mean that the curves in the pants decomposition lie above any short curve in $v_{n}^{-}$and any of the first $n$ curves in our 
list that overlap $W$. This will allow us to establish Properties (1)-(5) in Lemma 5.5. We now make this precise.

Let $c_{n}^{+}$contain every simple closed curve component of $v_{n}^{+}$. If $W$ is a subsurface which supports a conformal structure in $v_{n}^{+}$, let $\left.c_{n}^{+}\right|_{W}$ be a minimal length pants decomposition of $W$ which intersects every component of $b^{-} \cup e_{n}^{-}$which overlaps $W$. If the subsurface $W$ is the support of a lamination in $v_{n}^{+}$, let $\left.c_{n}^{+}\right|_{W}$ be a pants decomposition of $W$ of length at most $L_{1}$ in $N_{\rho_{n}}$, so that each curve in $\left.c_{n}^{+}\right|_{W}$ lies above every curve in $\alpha^{-1}([0, n]) \cup e_{n}^{-}$which overlaps $W$ (see Lemma 2.9 for the existence of such a pants decomposition).

Similarly, we define $c_{n}^{-}$so that it contains every closed curve component of $v_{n}^{-}$. If $W$ is a subsurface which supports a conformal structure in $v_{n}^{-}$, let $\left.c_{n}^{-}\right|_{W}$ be a minimal length pants decomposition of $W$ which intersects every component of $b^{+} \cup c_{n}^{+}$which overlaps $W$. If the subsurface $W$ is the support of a lamination in $v_{n}^{-}$, let $\left.c_{n}^{-}\right|_{W}$ be a pants decomposition of $W$ of length at most $L_{1}$ so that each curve in $\left.c_{n}^{-}\right|_{W}$ lies below every curve in $\alpha^{-1}([0, n]) \cup c_{n}^{+}$which overlaps $W$ (again see Lemma 2.9).

As in the quasifuchsian case, $\left\{\ell_{\rho_{n}}\left(c_{n}^{+} \cup c_{n}^{-}\right)\right\}$is bounded and $\left\{c_{n}^{ \pm}\right\}$has properties (3), (4) and (5) of Lemma 5.5.

Let $Y \subseteq S$ be an essential subsurface. If $Y$ lies in a subsurface $W$ which supports a conformal structure in $v_{n}^{\beta}$. Then, as in the proof of inequality (5.1), Lemma 2.1 implies that

$$
d_{Y}\left(v_{n}^{\beta}, c_{n}^{\beta}\right) \leq K
$$

for large enough $n$ as long as $Y$ is not a component of $\operatorname{collar}\left(c_{n}^{\beta}\right)$. If a simple closed curve component $p$ of $v_{n}^{\beta}$ intersects $Y$ essentially, then $p \subset c_{n}^{\beta}$ and $p$ is a closed curve without transversal in the base of the generalized marking $\mu\left(v_{n}^{\beta}\right)$ associated to $v_{n}^{\beta}$ (see section 2.2). Hence we have

$$
d_{Y}\left(v_{n}^{\beta}, c_{n}^{\beta}\right) \leq 2
$$

Finally, if $Y$ overlaps a subsurface $W$ which is the base surface of a lamination component of $v_{n}^{\beta}$, and $n \geq \alpha(d)$ for some $d \subset \partial Y$ that intersects $W$ essentially, Theorem 2.8 then implies that

$$
d_{Y}\left(v_{n}^{\beta}, c_{n}^{\beta}\right) \leq D
$$

Notice that in this last case we need $\partial Y \neq \emptyset$. We have proved that $\left\{c_{n}^{ \pm}\right\}$satisfies property (2). Since $v_{n}^{\beta}$ is never an ending lamination supported on all of $S, v_{n}^{\beta}$ contains either a closed curve or a conformal structure, so Property (1) holds as well. Lemma 5.5 then allows us to complete the proof in the case that $v_{n}^{\beta}$ is never an ending lamination supported on all of $S$.

To complete the proof, we consider the case where there exists $\beta_{0} \in\{ \pm\}$ such that for all $n, v_{n}^{\beta_{0}}$ is a lamination supported on all of $S$. Notice that in this case, Property (1) cannot hold, so we will need to again alter the construction somewhat. 
If $v_{n}^{\beta}$ is not a lamination supported on all of $S$, then we choose $c_{n}^{\beta}$ exactly as above. If $v_{n}^{\beta}$ is a lamination supported on all of $S$, then, by Minsky's Lipschitz Model Theorem [31], there exists $L_{0}$ and a tight geodesic $g_{n}$ joining $\mu\left(v_{n}^{+}\right)$to $\mu\left(v_{n}^{-}\right)$such that for any vertex $d$ of $g_{n}$, we have $\ell_{\rho_{n}}(d) \leq L_{0}$. Since $\left\{v_{n}^{ \pm}\right\}$bounds projections, there exists $K>0$ and a vertex $d_{n}$ of $g_{n}$, such that $d_{S}\left(d_{n}, \mu\right) \leq K$. Minsky's Lipschitz Model Theorem [31] again implies that there exists a pants decomposition $c_{n}^{\beta}$ of $S$ containing a vertex of $g_{n}$ between $d_{n}$ and $\mu\left(v_{n}^{\beta}\right)$ such that $\ell_{\rho_{n}}\left(c_{n}^{\beta}\right) \leq L_{1}$, and any curve in $c_{n}^{\beta}$ lies above every curve in $\alpha^{-1}([0, n]) \cup e_{n}^{-}$if $\beta=+$ and any curve in $c_{n}^{\beta}$ lies below every curve in $\alpha^{-1}([0, n]) \cup c_{n}^{+}$if $\beta=-$.

One then verifies properties (2)-(5) of Lemma 5.5 just as above. Property (1) was only used to prove condition (a), i.e. that every geodesic in $\mathscr{C}(S)$ joining $c_{n}^{+}$to $c_{n}^{-}$passes through a fixed bounded set. However, in the case that $v_{n}^{\beta_{0}}$ is always a lamination supported on all of $S$, it follows directly from our construction and the hyperbolicity of the curve complex ([26]) that any geodesic joining $c_{n}^{+}$to $c_{n}^{-}$passes within a uniformly bounded distance of $\mu$. This completes the proof of Proposition 5.1 in our final case.

\section{BOUNDED PROJECTIONS IMPLIES CONVERGENCE}

In this section we prove that if a sequence of Kleinian surface groups admits a pair of sequences of multicurves of uniformly bounded length which bounds projections, then it has a convergent subsequence. We first handle the case where the sequence of multicurves does not have any combinatorial wrapped parabolics, and then handle the general case by applying an argument motivated by work of Kerckhoff and Thurston [21].

6.1. In the absence of combinatorial wrapped parabolics. We recall that if a sequence $\left\{c_{n}^{ \pm}\right\}$of pairs of multicurves bounds projections and there are no combinatorial wrapped parabolics, then for any curve $d$ and complete marking $\mu$ there exists $\beta(d)$ such that $\left\{m\left(c_{n}^{\beta(d)}, d, \mu\right)\right\}$ is eventually bounded.

Proposition 6.1. Suppose that $\left\{\rho_{n}\right\}$ is a sequence in $A H(S)$ and there exists a sequence $\left\{c_{n}^{ \pm}\right\}$of pairs of multicurves such that $\left\{\ell_{\rho_{n}}\left(c_{n}^{+} \cup c_{n}^{-}\right)\right\}$is bounded and $\left\{c_{n}^{ \pm}\right\}$bounds projections and has no combinatorial wrapped parabolics. Then $\left\{\rho_{n}\right\}$ has a convergent subsequence.

Remark: Notice that any bounded sequence in $Q F(S)$ will admit bounded length multicurves which bound projections (any pair of filling pants decompositions will work). Therefore, we can only conclude that there exist a convergent subsequence.

Moreover, unlike in the end invariants case, a sequence of wrapped multicurves which bounds projections need not predict all the parabolics in the limit and need not predict which parabolics wrap. Notice that if $\left\{\rho_{n}\right\}$ converges and $c^{+}$and $c^{-}$ is any pair of filling multicurves, then the constant sequence $\left\{c_{n}^{ \pm}=c^{ \pm}\right\}$will be a sequence of pairs of bounded length multicurves bounding projections. In this case, $\left\{c_{n}^{ \pm}\right\}$does not predict any parabolics or ending laminations. 
Proof. We first show that, after passing to a subsequence $\left\{\rho_{j}\right\}$, there exists a fixed pants decomposition which has bounded length in all $N_{\rho_{j}}$.

Lemma 6.2. Suppose that $\left\{\rho_{n}\right\}$ is a sequence in $A H(S)$ and consider a sequence $\left\{c_{n}^{ \pm}\right\}$of pairs of multicurves which bound projections without combinatorial wrapped parabolics. If $\left\{\ell_{\rho_{n}}\left(c_{n}^{+} \cup c_{n}^{-}\right)\right\}$is bounded, then there exists a subsequence $\left\{\rho_{j}\right\}$ and a pants decomposition $r$ of $S$, so that $\left\{\ell_{\rho_{j}}(r)\right\}$ is a bounded sequence.

Proof. By assumption, there is a bounded region $\mathscr{B}$ in $\mathscr{C}(S)$ such that any geodesic joining $c_{n}^{+}$to $c_{n}^{-}$intersects $\mathscr{B}$. For all $n$, let $b_{n}$ be a curve on the geodesic joining $c_{n}^{+}$to $c_{n}^{-}$which is contained in $\mathscr{B}$. By Theorem 2.6, there exists $D$ and $L$ such that, for all $n$, there exists a curve $a_{n} \in \mathscr{C}(S)$ such that $d\left(a_{n}, b_{n}\right) \leq D$ and $\ell_{\rho_{n}}\left(a_{n}\right) \leq L$.

If $\left\{a_{n}\right\}$ admits a constant subsequence, then we pass to the appropriate subsequence of $\left\{\rho_{n}\right\}$ and the constant curve is the first curve in our pants decomposition $r$.

If not, by Lemma 2.3, there is a subsurface $Y$ such that $d_{Y}\left(a_{n}, \mu\right)$ diverges. Since $a_{n}$ is contained in a bounded region of $\mathscr{C}(S), Y$ is a proper subsurface of $S$. By assumption, there exists $\beta \in\{ \pm\}$, so that $d_{Y}\left(c_{n}^{\beta}, \mu\right)$ is bounded, hence $d_{Y}\left(c_{n}^{\beta}, a_{n}\right) \rightarrow \infty$. Then, by Theorem $2.5, \ell_{\rho_{n}}(\partial Y) \rightarrow 0$. In this case, the components of $\partial Y$ are the first curves in $r$.

We now assume that $r$ is non-empty and not yet a pants decomposition. We apply a mild variation of the above argument to show that we can enlarge $r$. This will eventually complete the proof. Let $W$ be a component of $S-r$ which is not a thricepunctured sphere. Since $r$ has uniformly bounded length, one may use Lemma 2.11 to find, for all $n$, a curve $b_{n} \in \mathscr{C}(W)$ so that $\ell_{\rho_{n}}\left(b_{n}\right)$ is uniformly bounded. By assumption, there exists $\beta \in\{ \pm\}$ so that $d_{W}\left(c_{n}^{\beta}, \mu\right)$ is eventually bounded. Let $L \geq L_{0}$ be an upper bound for both $\left\{\ell_{\rho_{n}}\left(c_{n}^{\beta}\right)\right\}$ and $\left\{\ell_{\rho_{n}}\left(b_{n}\right)\right\}$ (where $L_{0}=L_{0}(S)$ is the constant from Theorem 2.6). Theorem 2.6 implies that there exists $D=D(S, L)$ such that either $\operatorname{diam}\left(\pi_{W}\left(\mathscr{C}\left(\rho_{n}, L\right)\right) \leq D\right.$ or $d_{W}\left(c_{n}^{\beta}, \mathscr{C}\left(W, L, \rho_{n}\right)\right) \leq D$ for all $n$ (since $\left.c_{n}^{\beta} \in \mathscr{C}(\rho, L)\right)$. In the first case, $d_{W}\left(b_{n}, c_{n}^{\beta}\right) \leq D$, while in the second case there exists $a_{n} \in \mathscr{C}\left(W, L, \rho_{n}\right)$ such that $d_{W}\left(c_{n}^{\beta}, a_{n}\right) \leq D$. In the first case, we let $a_{n}=b_{n}$. Therefore, in either case, we have constructed a sequence $\left\{a_{n}\right\}$ in $\mathscr{C}(W)$ such that $\ell_{\rho_{n}}\left(a_{n}\right) \leq L$ and $d_{W}\left(c_{n}^{\beta}, a_{n}\right) \leq D$.

If $\left\{a_{n}\right\}$ admits a constant subsequence, then we pass to the appropriate subsequence of $\left\{\rho_{n}\right\}$ and add the constant curve to $r$. If not, by Lemma 2.3 there is a subsurface $Y$ such that $d_{Y}\left(a_{n}, \mu\right)$ diverges. Since $\left\{d_{W}\left(a_{n}, \mu\right)\right\}$ is eventually bounded, $Y$ is a proper subsurface of $W$. We can again argue, as in the third paragraph of the proof, that $d_{Y}\left(c_{n}^{\beta^{\prime}}, a_{n}\right) \rightarrow \infty$ for some $\beta^{\prime} \in\{ \pm\}$. By Theorem 2.5, $\ell_{\rho_{n}}(\partial Y) \rightarrow 0$. In this case, we may add $\partial Y-\partial W$ to $r$.

Next we construct, for every curve in $r$ a transversal which has bounded length in all $N_{\rho_{j}}$, perhaps after passage to a further subsequence. By Lemma 2.11, there are bounded length pants decompositions $r_{j}^{+}$and $r_{j}^{-}$in $N_{\rho_{j}}$ containing $c_{j}^{+}$and $c_{j}^{-}$, respectively. We may pass to a subsequence so that $r \cap r_{j}^{+}$and $r \cap r_{j}^{-}$are both 
constant. (Here, we use $r \cap r_{j}^{\beta}$ as shorthand for the collection of curves which lie in both $r$ and $r_{j}^{\beta}$.)

Let $d$ be a curve in $r$. There exists a choice of sign $\beta=\beta(d) \in\{ \pm\}$ so that $m\left(c_{j}^{\beta}, d, \mu\right)$ is bounded for all $j$, perhaps after again passing to a subsequence. In particular, this implies that $d$ does not lie in $r_{j}^{\beta}$ (since $d$ must intersect $c_{j}^{\beta}$ if $m\left(c_{j}^{\beta}, d, \mu\right)$ is finite). Let $G=G(d)$ be the subsurface of $S-\left(r \cap r_{j}^{\beta}\right)$ which contains $d$.

Let $H_{j}=H_{j}(d)$ be a hierarchy in $\mathscr{C}(G)$ joining $r_{j}^{\beta} \cap G$ and $r \cap G$. Here we regard both $r_{j}^{\beta} \cap G$ and $r \cap G$ as markings without transversals. (Hierarchies are defined and discussed extensively in Masur-Minsky [27].)

Let $\sigma_{j} \in A H(G)$ be the unique Kleinian group so that $r_{j}^{\beta} \cap G$ is the collection of upward-pointing parabolic and $r \cap G$ is the collection of downward-pointing parabolics. Let $X_{j}=N_{\sigma_{j}}=\mathbf{H}^{3} / \sigma_{j}\left(\pi_{1}(G)\right)$. (The hyperbolic manifold $X_{j}$ is called a maximal cusp, see Keen-Maskit-Series [20] for a proof of the existence and uniqueness of $X_{j}$. The existence also follows from Thurston's Geometrization Theorem for pared manifolds, see Morgan [32].) Notice that $r_{j}^{\beta} \cap G$ and $r \cap G$ are the end invariants of $X_{j}$.

Let $M_{j}$ be the model manifold associated to the hierarchy $H_{j}$. (The construction of a model manifold associated to a hierarchy is carried out in Minsky [31, Sec. 8].) The Bilipschitz Model Manifold Theorem [12] guarantees that there exists a bilipschitz homeomorphism $g_{j}: M_{j} \rightarrow X_{j}$.

The hierarchy $H_{j}$ is a family of tight geodesics. The base tight geodesic lies in $\mathscr{C}(G)$ and joins $r_{j}^{\beta} \cap G$ to $r \cap G$. Theorem 2.7 implies that there is a uniform upper bound on the length $\ell_{\rho_{j}}(c)$ of any curve $c$ which is contained in a vertex of the base tight geodesic. Then $H_{j}$ is constructed iteratively by appending tight geodesics in curve complexes of subsurfaces of $G$ which join vertices in previously added tight geodesics. Since this process terminates after a finite (bounded) number of steps, Theorem 2.7 implies that there is a uniform upper bound on the length $\ell_{\rho_{j}}(c)$ of any curve $c$ contained in a vertex in the hierarchy $H_{j}$.

The model manifold $M_{j}$ is constructed from blocks of two isometry types, one homeomorphic to the product of a one-holed torus and the interval and the other homeomorphic to the product of a four-holed sphere and the interval, tubes, which are isometric to Margulis regions in hyperbolic 3-manifolds, and a finite number of boundary blocks. Each block is associated to an edge of a geodesic in the curve complex of either a one-holed torus or a four-holed sphere. These geodesics are called 4-geodesics.

Let $\hat{M}_{j}$ be obtained from $M_{j}$ by removing the tubes and the boundary blocks. So, $\hat{M}_{j}$ consists entirely of blocks. Since all the vertices have uniformly bounded length, the techniques of section 10 of Minsky [31] (in particular, see Steps 0-5) imply that there exists a $K$-Lipschitz map $h_{j}: \hat{M}_{j} \rightarrow N_{\rho_{j}}$ where $K$ depends only on $S$ and the uniform bound on the lengths of the curves in $H_{j}$ obtained from Theorem 2.7 . 
Let $A_{d, j}$ be the intersection of $\hat{M}_{j}$ with $U(d)$, the tube in $M_{j}$ associated to $d$. The annulus $A_{d, j}$ is made up of $s_{j}(d)+1$ bounded geometry annuli where $s_{j}(d)$ is the number of edges of 4-geodesics in $H_{j}$ whose domains contain $d$ in their boundary. The arguments in Theorem 9.11 of Minsky [31] imply that

$$
s_{j}(d) \leq C\left(\sup _{d \in \partial Y, Y \neq \operatorname{collar}(d)} d_{Y}\left(r, r_{j}^{\beta}\right)\right)^{a}
$$

for uniform constants $C$ and $a$. However,

$$
\sup _{d \in \partial Y, Y \neq \operatorname{collar}(d)} d_{Y}\left(r, r_{j}^{\beta}\right) \leq m\left(c_{j}^{\beta}, d, \mu\right)+\sup _{d \in \partial Y, Y \neq \operatorname{collar}(d)} d_{Y}(r, \mu) .
$$

The first term on the right hand side is uniformly bounded by assumption, while the second term is finite and independent of $j$. Therefore, $s_{j}(d)$ is bounded, which implies that the geometry of $A_{d, j}$ is uniformly bounded.

It follows that there is an essential curve $t_{d, j}$ of uniformly bounded length in $\partial \hat{M}_{j}$ which is disjoint from the boundaries of the annuli associated to components of $r \cap G-d$ and intersects $U(d)$ minimally, i.e. in two arcs if $U(d)$ separates the component of $G-(r \cap G)$ it is contained in and in one arc otherwise. The image $g_{j}\left(t_{d, j}\right)$ in $X_{j}$ is a curve, of uniformly bounded length, which lies above the cusp associated to $d$. Theorem 2.8 then implies that $d_{Y}\left(t_{j, d}, r_{j}^{\beta}\right)$ is uniformly bounded when $d \subset \partial Y$. Since, $m\left(c_{j}^{\beta}, \mu\right)$ is uniformly bounded and

$$
\left|d_{Y}\left(c_{j}^{\beta}, \mu\right)-d_{Y}\left(r_{j}^{\beta}, \mu\right)\right| \leq 1,
$$

we see that $d_{Y}\left(t_{j, d}, \mu\right)$ is uniformly bounded for any subsurface $Y \subset S$ whose boundary contains $d$. Since any two curves which are disjoint from $r \cap G-d$ and intersect $d$ minimally differ, up to homotopy, by a power of a Dehn twist in $U(d)$, there are only finitely many possibilities for $t_{j, d}$. Therefore, we may pass to a subsequence so that $t_{j, d}=t_{d}$ for a fixed curve $t_{d}$. The length $\ell_{\rho_{j}}\left(t_{d}\right)$ is uniformly bounded, since $h_{j}\left(t_{d}\right)$ is a bounded length representative of $t_{d}$ in $N_{\rho_{j}}$.

We have found a pants decomposition $r$ and a system of transversals $\left\{t_{d}\right\}_{d \in r}$ such that all curves in $r$ and their transversals have uniformly bounded length in $\left\{N_{\rho_{j}}\right\}$. It then follows from Thurston's Double Limit Theorem [37, 34] that $\left\{\rho_{j}\right\}$ has a convergent subsequence.

Remark: With a little more care, one may use this same argument to find a surface in $N_{\rho_{j}}$, for all large enough $j$, where $r$ and $\left\{t_{d}\right\}_{d \in r}$ have uniformly bounded length. One can then verify convergence up to subsequence more directly.

6.2. The general case. We now use ideas based on work of Kerckhoff and Thurston [21] to handle the general case.

Proposition 6.3. Suppose that $\left\{\rho_{n}\right\}$ is a sequence in $A H(S)$ and there exists a sequence of pairs, $\left\{c_{n}^{ \pm}\right\}$, of multicurves such that $\left\{\ell_{\rho_{n}}\left(c_{n}^{+} \cup c_{n}^{-}\right)\right\}$is bounded and $\left\{c_{n}^{ \pm}\right\}$bounds projections. Then $\left\{\rho_{n}\right\}$ has a convergent subsequence. 
Proof. Let $q$ be the set of combinatorial wrapped parabolics for $\left\{c_{n}^{ \pm}\right\}$. We recall that $d \in q$ if and only if $\left\{m^{n a}\left(c_{n}^{+}, d, \mu\right)\right\}$ and $\left\{m^{n a}\left(c_{n}^{-}, d, \mu\right)\right\}$ are both eventually bounded and there exists $w=w(d) \in \mathbf{Z}$ and a sequence $\left\{s_{n}=s_{n}(d)\right\} \subset \mathbb{Z}$ such that $\lim \left|s_{n}\right|=\infty$ and both $\left\{d_{Y}\left(\left(D_{Y}^{s_{n} w}\left(c_{n}^{+}\right), \mu\right)\right\}\right.$ and $\left\{d_{Y}\left(D_{Y}^{s_{n}(w-1)}\left(c_{n}^{-}\right), \mu\right)\right\}$ are eventually bounded when $Y=\operatorname{collar}(d)$.

Notice that if $q$ is empty, then Proposition 6.3 follows from Proposition 6.1. We first observe that $q$ is a multicurve.

Lemma 6.4. The set $q$ of combinatorial wrapping parabolics is a multicurve.

Proof. Suppose that $q$ contains intersecting curves $c$ and $d$, and let $Y=\operatorname{collar}(c)$ and $Z=\operatorname{collar}(d)$. Lemma 2.2 then implies that

$$
\min \left\{d_{Y}\left(\partial Z, c_{n}^{+}\right), d_{Z}\left(\partial Y, c_{n}^{+}\right)\right\} \leq 10
$$

which contradicts the fact that both $d_{Y}\left(c_{n}^{+}, \mu\right) \rightarrow \infty$ and $d_{Z}\left(c_{n}^{+}, \mu\right) \rightarrow \infty$.

Let $Q=\bigcup_{q_{i} \in q} Q_{i}=\operatorname{collar}\left(q_{i}\right)$ be a regular neighborhood of $q$ and consider the diffeomorphisms

$$
\Phi_{n}^{+}=\Pi_{q_{i} \in q} D_{Q_{i}}^{s_{n}\left(q_{i}\right) w\left(q_{i}\right)} \text { and } \Phi_{n}^{-}=\prod_{q_{i} \in q} D_{Q_{i}}^{s_{n}\left(q_{i}\right)\left(w\left(q_{i}\right)-1\right)}
$$

where $D_{Q_{i}}$ is the right Dehn twist about the annulus $Q_{i}$.

Lemma 6.5. The pairs of sequences $\left\{\Phi_{n}^{+}\left(c_{n}^{ \pm}\right)\right\}$and $\left\{\Phi_{n}^{-}\left(c_{n}^{ \pm}\right)\right\}$both bound projections and have no combinatorial wrapped parabolics.

Proof. We first prove that $\left\{\Phi_{n}^{+}\left(c_{n}^{ \pm}\right)\right\}$bounds projections.

Let $d$ be a curve in $q$. Since $\left\{c_{n}^{ \pm}\right\}$bounds projections, $d$ lies a uniformly bounded distance from any geodesic joining $c_{n}^{+}$to $c_{n}^{-}$. Notice that if $c \in \mathscr{C}(S)$, then $d_{S}\left(d, \Phi_{n}^{+}(c)\right)=d_{S}(d, c)$. Since any geodesic joining $\Phi_{n}^{+}\left(c_{n}^{+}\right)$to $\Phi_{n}^{+}\left(c_{n}^{-}\right)$is the image under $\Phi_{n}^{+}$of a geodesic joining $c_{n}^{+}$to $c_{n}^{-}$, it follows that $d$ also lies a uniformly bounded distance from any geodesic joining $\Phi_{n}^{+}\left(c_{n}^{+}\right)$to $\Phi_{n}^{+}\left(c_{n}^{-}\right)$. Hence the pair of sequence $\left\{\Phi_{n}^{+}\left(c_{n}^{+}\right)\right\}$and $\left\{\Phi_{n}^{+}\left(c_{n}^{-}\right)\right\}$satisfies condition (a) in the definition of bounding projections.

Let $d \subset S$ be a simple closed curve which is not a component of $q$. If $d$ does not cross $q$ then $m\left(c_{n}^{ \pm}, d, \mu\right)=m\left(\Phi_{n}^{+}\left(c_{n}^{ \pm}\right), d, \mu\right)$ for all $n$. Since $\left\{c_{n}^{ \pm}\right\}$bounds projections and $d$ is not a combinatorial wrapping parabolic, it follows that there exists $\beta \in\{ \pm\}$ such that $\left\{m\left(\Phi_{n}^{+}\left(c_{n}^{\beta}\right), d, \mu\right)\right\}$ is eventually bounded.

If $d$ crosses a component $q_{i}$ of $q$, it follows from the definition of $\Phi_{n}^{ \pm}$that $d_{Q_{i}}\left(d, \Phi_{n}^{+}\left(c_{n}^{-}\right)\right) \longrightarrow \infty$ where $Q_{i}$ is the collar neighborhood of $q_{i}$. Lemma 2.2 then implies that if $n$ is large enough, then $d_{Y}\left(q_{i}, \Phi_{n}^{+}\left(c_{n}^{-}\right)\right) \leq 4$ for any subsurface $Y$ whose boundary contains $d$. Thus, again if $n$ is large enough, by Lemma 2.1,

$$
d_{Y}\left(\mu, \Phi_{n}^{+}\left(c_{n}^{-}\right)\right) \leq d_{Y}\left(\mu, q_{i}\right)+d_{Y}\left(q_{i}, \Phi_{n}^{+}\left(c_{n}^{-}\right)\right) \leq 1+2 i\left(q_{i}, \mu\right)+4=5+2 i\left(q_{i}, \mu\right)
$$

for any subsurface $Y$ whose boundary contains $d$. Therefore, $\left\{m\left(\Phi_{n}^{+}\left(c_{n}^{-}\right), d, \mu\right)\right\}$ is eventually bounded.

If $d=q_{i}$ is a component of $Q$, then $m^{n a}\left(c_{n}^{+}, q_{i}, \mu\right)=m^{n a}\left(\Phi_{n}^{+}\left(c_{n}^{+}\right), q_{i}, \mu\right)$ for all $n$, so $\left\{m^{n a}\left(\Phi_{n}^{+}\left(c_{n}^{+}\right), q_{i}, \mu\right)\right\}$ is eventually bounded. By definition of $\Phi_{n}^{+},\left\{d_{Q_{i}}\left(\Phi_{n}^{+}\left(c_{n}^{+}\right), \mu\right)\right.$ is eventually bounded. Therefore, $\left\{m\left(\Phi_{n}^{+}\left(c_{n}^{+}\right), q_{i}, \mu\right)\right\}$ is eventually bounded 
We have proved that for any simple closed curve $d \subset S$ there is $\beta$ such that $m\left(\Phi_{n}^{+}\left(c_{n}^{\beta}\right), d, \mu\right)$ is eventually bounded. This completes the proof that the pair $\left\{\Phi_{n}^{+}\left(c_{n}^{ \pm}\right)\right\}$bounds projections without combinatorial wrapped parabolics.

The proof that the sequences of pairs $\left\{\Phi_{n}^{-}\left(c_{n}^{ \pm}\right)\right\}$bounds projections without combinatorial wrapped parabolics is analogous.

For each $n$, consider the representations

$$
\rho_{n}^{+}=\rho_{n} \circ\left(\Phi_{n}^{+}\right)_{*}^{-1} \text { and } \rho_{n}^{-}=\rho_{n} \circ\left(\Phi_{n}^{-}\right)_{*}^{-1} .
$$

By construction, the sequences $\left\{\ell_{\rho_{n}^{\beta}}\left(\Phi_{n}^{\beta}\left(c_{n}^{ \pm}\right)\right)\right\}=\left\{\ell_{\rho_{n}}\left(c_{n}^{ \pm}\right)\right\}$are uniformly bounded for any $\beta \in\{ \pm\}$. Lemma 6.5 implies that $\left\{\Phi_{n}^{+}\left(c_{n}^{ \pm}\right)\right\}$and $\left\{\Phi_{n}^{-}\left(c_{n}^{ \pm}\right)\right\}$both bound projections and have no combinatorial wrapped parabolics, so Proposition $6.1 \mathrm{im}-$ plies that we may pass to a subsequence so that both $\left\{\rho_{n}^{+}\right\}$and $\left\{\rho_{n}^{-}\right\}$converge to discrete, faithful representations $\rho^{+}$and $\rho^{-}$.

Extend $q$ to a pants decomposition $p$ of $S$. If $d \in p$, then $\ell_{\rho_{n}}(d)=\ell_{\rho_{n}^{+}}(d)$ for all $n$, so $\left\{\ell_{\rho_{n}}(d)\right\}$ is bounded. Let $\hat{p}$ be a maximal collection of transversals to the elements of $p$ (i.e. each element of $\hat{p}$ intersects exactly one element of $p$ and does so minimally). If $t \in \hat{p}$ is a transversal to an element of $p-q$, then again $\ell_{\rho_{n}}(t)=\ell_{\rho_{n}^{+}}(t)$ for all $n$, so $\left\{\ell_{\rho_{n}}(t)\right\}$ is bounded

Lemma 6.6. If $t \in \hat{p}$ is a transversal to an element $d$ of $q$, then $\left\{\ell_{\rho_{n}}(t)\right\}$ is bounded.

Proof. We show that any subsequence of $\left\{\rho_{n}\right\}$ contains a further subsequence such that $\left\{\rho_{n}(t)\right\}$ converges. Our result then follows immediately.

We first pass to a subsequence, and fix a specific representative in each conjugacy class, so that $\left\{\rho_{n}^{+}=\rho_{n} \circ\left(\Phi_{n}^{+}\right)_{*}^{-1}\right\}$ converges as a sequence of representations into $\operatorname{PSL}(2, \mathbb{C})$. (The existence of such a subsequence follows from Lemma 6.5 and Proposition 6.1.) Since $\Phi_{n}^{+}$and $\Phi_{n}^{-}$restrict to the identity on $S-Q$, and $\left\{\rho_{n}^{-}\right\}$ has a convergent subsequence in $A H(S)$ (again by Lemma 6.5 and Proposition 6.1), we may pass to a further subsequence so that $\left\{\rho_{n}^{-}\right\}$also converges as a sequence of representations into $\operatorname{PSL}(2, \mathbb{C})$.

Let us first consider the case where $t$ intersects $d$ exactly once. Then, with an appropriate choice of basepoint for $\pi_{1}(S)$, we have

$$
\rho_{n}^{-}(t)=\rho_{n}\left(d^{(w(d)-1) s_{n}} t\right)=\rho_{n}^{+}\left(d^{-s_{n}} t\right)
$$

so $\rho_{n}^{+}\left(d^{-s_{n}}\right)=\rho_{n}^{-}(t) \rho_{n}^{+}(t)^{-1}$. Since $\left\{\rho_{n}^{-}(t)\right\}$ and $\left\{\rho_{n}^{+}(t)\right\}$ both converge we immediately conclude that $\left\{\rho_{n}^{+}\left(d^{s_{n}}\right)=\rho_{n}\left(d^{s_{n}}\right)\right\}$ and $\left\{\rho_{n}(t)=\rho_{n}\left(d^{-w(d) s_{n}}\right) \rho_{n}^{+}(t)\right\}$ converge.

In the slightly more complicated second case where $t$ intersects $d$ twice, we argue by contradiction. We first homotope $t$ so that the two points of $t \cap d$ coincide. Then $t$ is the concatenation of two loops $a$ and $b$ which are freely homotopic to curves that are disjoint from $d$ and $\rho_{n}(t)=\rho_{n}(a b)$. With an appropriate choice of basepoint for $\pi_{1}(S)$, we have

$$
\rho_{n}(a)=\rho_{n}^{+}(a)=\rho_{n}^{-}(a), \quad \rho_{n}(d)=\rho_{n}^{+}(d)=\rho_{n}^{-}(d),
$$


and

$$
\rho_{n}^{-}(b)=\rho_{n}\left(d^{(w(d)-1) s_{n}} b d^{-(w(d)-1) s_{n}}\right)=\rho_{n}^{+}\left(d^{-s_{n}} b d^{s_{n}}\right) .
$$

Suppose that $\left\{\rho_{n}\left(d^{s_{n}}\right)=\rho_{n}^{+}\left(d^{s_{n}}\right\}\right.$ exits every compact subset of PSL $(2, \mathbb{C})$ and pick $p \in \mathbb{H}^{3}$. Since the fixed points of $\rho_{n}^{+}(d)$ and $\rho_{n}^{+}(b)$ converge to distinct sets (i.e. the fixed points of $\rho^{+}(d)$ and $\left.\rho^{+}(b)\right), \rho_{n}^{+}\left(d^{s_{n}}\right)(p)$ converges to a point in $\partial \mathbb{H}^{3}$ disjoint from the fixed point set of $\rho^{+}(b)$. It follows that

$$
d\left(\rho_{n}^{+}\left(b d^{s_{n}}\right)(p), \rho_{n}^{+}\left(d^{s_{n}}\right)(p)\right) \rightarrow \infty .
$$

Applying $\rho_{n}^{+}\left(d^{-s_{n}}\right)$ to each term we see that

$$
d\left(\rho_{n}^{+}\left(d^{-s_{n}} b d^{s_{n}}\right)(p), p\right) \rightarrow \infty,
$$

which contradicts the fact that $\left\{\rho_{n}^{-}(b)=\rho_{n}^{+}\left(d^{-s_{n}} b d^{s_{n}}\right)\right\}$ converges. Therefore, a subsequence of $\left\{\rho_{n}\left(d^{s_{n}}\right)\right\}$ converges. It follows that, with the same subsequence, $\left\{\rho_{n}(b)=\rho_{n}\left(d^{-w(d) s_{n}}\right) \rho_{n}^{+}(b) \rho_{n}\left(d^{w(d) s_{n}}\right)\right\}$ and $\left\{\rho_{n}(t)=\rho_{n}(a b)\right\}$ both converge. (For a related argument see Anderson-Lecuire [4, Claim 7.1].) This completes the proof.

We have exhibited a pants decomposition and a complete collection of transversals all of whose images under $\rho_{n}$ have bounded length. Therefore, Thurston's Double Limit Theorem [37, 34] again implies that $\left\{\rho_{n}\right\}$ has a convergent subsequence.

\section{CONCLUSION}

We will now assemble the previous results to establish Theorems 1.1, 1.2 and 1.3. Let $S$ be a compact, orientable surface and let $\left\{\rho_{n}\right\}$ be a sequence in $A H(S)$ with end invariants $\left\{v_{n}^{ \pm}\right\}$.

Proof of Theorem 1.1: If $\left\{v_{n}^{ \pm}\right\}$has a subsequence $\left\{v_{j}^{ \pm}\right\}$which bounds projections, then Proposition 5.1 implies that there exists a further subsequence, still called $\left\{\rho_{j}\right\}$, and a sequence $\left\{c_{j}^{ \pm}\right\}$of pairs of multi-curves such that $\left\{\ell_{\rho_{j}}\left(c_{j}^{+} \cup c_{j}^{-}\right)\right\}$is bounded and $\left\{c_{j}^{ \pm}\right\}$bounds projections. Theorem 6.3 then implies that $\left\{\rho_{j}\right\}$, and hence $\left\{\rho_{n}\right\}$, has a convergent subsequence. On the other hand, if $\left\{\rho_{n}\right\}$ has a convergent sequence, it follows immediately from Theorem 4.1 that some subsequence of $\left\{v_{n}^{ \pm}\right\}$bounds projections.

Theorem 1.2 is precisely the second part of Theorem 4.1.

Proof of Theorem 1.3: Theorem 6.3 implies that if there exists a sequence $\left\{c_{n}^{ \pm}\right\}$of pairs of multi-curves such that $\left\{\ell_{\rho_{n}}\left(c_{n}^{+} \cup c_{n}^{-}\right)\right\}$is bounded and $\left\{c_{n}^{ \pm}\right\}$bounds projections, then $\left\{\rho_{n}\right\}$ has a convergent subsequence. On the other hand, if $\left\{\rho_{n}\right\}$ has a convergent subsequence $\left\{\rho_{j}\right\}$, then we may simply pick any filling pair $c^{ \pm}$of multicurves and set $c_{j}^{ \pm}=c^{ \pm}$for all $j$. Then, since $\left\{\rho_{j}\right\}$ is convergent, $\left\{\ell_{\rho_{j}}\left(c_{j}^{+} \cup c_{j}^{-}\right)\right\}$is bounded and $\left\{c_{j}^{ \pm}\right\}$bounds projections 


\section{REFERENCES}

[1] J.W. Anderson and R.D. Canary, "Algebraic limits of Kleinian groups which rearrange the pages of a book," Invent. Math. 126 (1996), 205-214.

[2] J.W. Anderson and R. Canary, "Cores of hyperbolic 3-manifolds and limits of Kleinian groups," Amer. J. Math. 118 (1996), 745-779.

[3] J.W. Anderson, R.D. Canary, and D. McCullough, "The topology of deformation spaces of Kleinian groups," Annals of Math. 152 (2000), 693-741.

[4] J.W. Anderson and C. Lecuire, "Strong convergence of Kleinian groups: the cracked eggshell," Comm. Math. Helv. 88 (2013), 813-857.

[5] J.A. Behrstock, ”Asymptotic Geometry of the Mapping Class Group and Teichmuller Space," Geometry \& Topology 10 (2006) 1523-1578.

[6] L. Bers, "On boundaries of Teichmüller spaces and Kleinian groups I," Annals of Math. 91 (1970), 570-600.

[7] L. Bers, "An inequality for Riemann surfaces," in Differential geometry and complex analysis, Springer-Verlag,1985, 87-93.

[8] F. Bonahon, "Bouts des variétés hyperboliques de dimension 3," Annals of Math. 124 (1986), 71-158.

[9] B. Bowditch, "Length bounds on curves arising from tight geodesics," G.A.F.A. 17 (2007), 1001-1042.

[10] J.F. Brock, K. Bromberg, R.D. Canary and Y.N.Minsky, "Local topology in deformation space of hyperbolic 3-manifolds," Geom. Top. 15 (2011), 1169-1224.

[11] J.F. Brock, K. Bromberg, R.D. Canary and Y.N.Minsky, "Convergence properties of ending invariants," Geom. Top. 17 (2013), 2877-2922.

[12] J.F. Brock, R.D. Canary and Y. Minsky, "The Classification of Kleinian Surface Groups II: The Ending Lamination Conjecture," Annals of Math., 176 (2012), 1-149.

[13] K. Bromberg and J. Holt, "Self-bumping of deformation spaces of hyperbolic 3-manifolds," $J$. Diff. Geom. 57 (2001), 47-65.

[14] P. Buser, Geometry and spectra of compact Riemann surfaces, Progress in mathematics 106, Birkhäuser, 1992.

[15] R.D. Canary, D.B.A. Epstein, P. Green, "Notes on notes of Thurston", in Analytical and Geometrical Aspects of Hyperbolic Space, London Math. Soc. Lecture Note Ser. 111 (1987), 3-92.

[16] D.B.A. Epstein, A. Marden and V. Markovic, "Quasiconformal homeomorphisms and the convex hull boundary," Annals of Math. 159 (2004), 305-336.

[17] R.A. Evan and J. Holt, "Non-wrapping of hyperbolic interval bundles," G.A.F.A. 18 (2008), 98-119.

[18] U. Hamenstadt, "Train tracks and the Gromov boundary of the complex of curves," in Spaces of Kleinian groups, London Math. Soc. Lecture Note Ser. 329 (2006), 187-207.

[19] T. Jørgensen, ”On discrete groups of Möbius transformations," Amer. J. Math. 98 (1976), 739749.

[20] L. Keen, B. Maskit and C. Series, "Geometric finiteness and uniqueness for groups with circle packing limit sets," J. reine und angew. Math. 436 (1993), 209-219.

[21] S.P. Kerckhoff and W.P. Thurston, "Non-continuity of the action of the modular group at Bers' boundary of Teichmüller space," Invent. Math. 100 (1990), 25-47.

[22] E. Klarreich, "The boundary at infinity of the curve complex and the relative Teichmüller space," preprint.

[23] R. Kulkarni and P. Shalen, "On Ahlfors' finiteness theorem," Adv. Math. 111 (1991), 155-169.

[24] J. Mangahas, "A recipe for short-word pseudo-Anosovs," Amer. J. Math. 135 (2013), 10871116.

[25] B. Maskit, Kleinian groups. Grundlehren der Mathematischen Wissenschaften [Fundamental Principles of Mathematical Sciences], 287 (1988). 
[26] H. A. Masur and Y. Minsky, "Geometry of the complex of curves I: Hyperbolicity," Invent. Math. 138 (1999), 103-149.

[27] H.A. Masur and Y.N. Minsky, "Geometry of the complex of curves II: Hierarchical structure," Geom. Funct. Anal. 10 (2000), 902-974.

[28] D. McCullough, "Compact submanifolds of 3-manifolds with boundary," Quart. J. Math. Oxford 37 (1986), 299-306.

[29] C. T. McMullen, "Complex earthquakes and Teichmüller theory," J. Amer. Math. Soc. 11 (1998), 283-320.

[30] Y. Minsky, "Kleinian groups and the complex of curves," Geom. and Top. 4 (2000), 117-148.

[31] Y. Minsky, "The classification of Kleinian surface groups I: models and bounds," Annals of Math. 171 (2010), 1-107.

[32] J. Morgan, "On Thurston's uniformization theorem for three-dimensional manifolds" in The Smith Conjecture, Pure Appl. Math. 112, Academic Press, 1984, 37-125.

[33] K. Ohshika, "Divergence, exotic convergence and self-bumping in quasi-Fuchsian spaces," preprint, available at arXiv:1010.0070.

[34] J.-P. Otal, Le théorème d'hyperbolisation pour les variétés fibrées de dimension 3. Astérisque 235 (1996).

[35] J.-P. Otal, "Les géodésiques fermées d'une variété hyperbolique en tant que nœuds”, in Kleinian groups and hyperbolic 3-manifolds, London Math. Soc. Lecture Note Ser. 299, Cambridge Univ. Press, 2003, 95-104.

[36] W. P. Thurston, The Geometry and Topology of Three-Manifolds, Princeton University course notes, available at: http://www.msri.org/publications/books/gt $3 \mathrm{~m} /$

[37] W.P. Thurston, "Hyperbolic structures on 3-manifolds, II: Surface groups and 3-manifolds which fiber over the circle," preprint, available at arXiv:math.GT/9801045. 